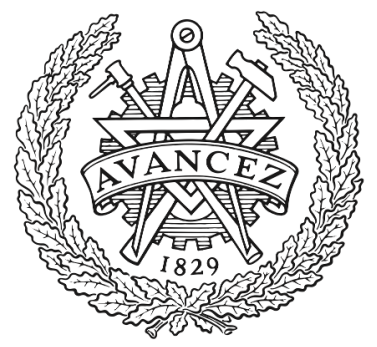

CHALMERS

UNIVERSITY OF TECHNOLOGY

\title{
Impact of ice aggregate parameters on microwave and sub-millimetre scattering properties
}

Downloaded from: https://research.chalmers.se, 2023-04-26 11:17 UTC

Citation for the original published paper (version of record):

Ekelund, R., Eriksson, P. (2019). Impact of ice aggregate parameters on microwave and sub-millimetre scattering properties. Journal of Quantitative Spectroscopy and Radiative Transfer, 224: 233-246. http://dx.doi.org/10.1016/j.jqsrt.2018.11.013

N.B. When citing this work, cite the original published paper. 


\title{
Impact of ice aggregate parameters on microwave and sub-millimetre scattering properties
}

\author{
Robin Ekelund ${ }^{\mathrm{a}}$, Patrick Eriksson ${ }^{\mathrm{a}}$ \\ ${ }^{a}$ Department of Space, Earth and Environment, Chalmers University of Technology, Gothenburg, Sweden.
}

\begin{abstract}
Microwave scattering properties for 1101 aggregates were calculated using DDA (Discrete Dipole Approximation), at three typical radar bands $(13.4,35.6,94.1 \mathrm{GHz})$ and three passive microwave frequencies $(183.31,325.15 \mathrm{and} 664 \mathrm{GHz})$. The aggregates were generated in a semi-physical stochastic fashion and are composed of hexagonal crystals of varying axis ratio, ranging from $1 / 15$ (plates) to 15 (columns). Horizontally aligned particles were assumed and scattering properties were assessed for zenith/nadir observations. Crystal axis ratio, number of crystals, effective density and aerodynamic area, were found to correlate with extinction and back-scattering efficiencies. However, the dependency between these variables and scattering properties vary between the frequencies. Interestingly, bulk extinction was found to have a relatively low sensitivity to particle shape at $664 \mathrm{GHz}$. Furthermore, extinction was found to be less shape sensitive than back-scattering. These results are promising for the sake of the upcoming Ice Cloud Imager (ICI) mission. In addition, for the considered set of aggregates, it is shown that both bulk extinction and back-scattering are more directly related to snow fall than ice water content. Triple frequency signatures were also calculated, which demonstrated clear dependence on constituent crystal axis ratio and conversely on aggregate effective density, in agreement with the literature.
\end{abstract}

Keywords: Ice hydrometeors, aggregates, single scattering properties, radar, microwaves, sub-millimetres.

\section{Introduction}

Frozen hydrometeors play an important role in the hydrological cycle, especially at mid- and high-latitudes where snow constitutes a large portion of the overall precipitation (Ellis et al., 2009). Also, improved observations of ice hydrometeors can lead to better weather predictions through data assimilation (Hou et al., 2014; Geer et al., 2017). Global observations of atmospheric ice are of high scientific and socio-economic interest, and global coverage is best achieved through satellite remote sensing measurements.

Radars and radiometers that operate at microwave wavelengths are suitable for retrieving ice mass thanks to their cloud penetration capability. The interaction between microwave radiation and particles depends strongly on the relationship between wavelength and particle size (Buehler et al., 2007). Current operational satellite microwave instruments have channels up to $190 \mathrm{GHz}$. With respect to ice hydrometeors, these sensors are thus mainly sensitive to precipitating particles, i.e. snow (Bühl et al., 2017). The Ice Cloud Imager (ICI) will extend the frequency coverage up to $664 \mathrm{GHz}$, into the sub-millimetre range, offering better sensitivity to ice crystals found in high-altitude clouds (Buehler et al., 2012). Satellite-based radars currently cover frequencies between 13 and $94 \mathrm{GHz}$. The current ones are the CloudSat cloud profiling radar (CPR), operating at W-band $(94 \mathrm{GHz})$, and the GPM dual-frequency phased-array precipitation radar (DPR), operating at $\mathrm{K}_{\mathrm{u}}$ and $\mathrm{K}_{\mathrm{a}}$-band $(13.6$ and $35.6 \mathrm{GHz}$, respectively).

The assimilation or inversion of such measurements by aforementioned systems requires accurate modelling of the light scattering by individual particles. The scattering is in turn affected by the refractive index (at given frequency) and the shape of the particle (Kim, 2006; Liu, 2008; Hong, 2007; Eriksson et al., 2015). Shape is considerably more complex and variable for ice hydrometeors than for liquid ones, which are approximately spheroidal. Small single ice crystals, as found in cirrus, are typically irregular in shape, but can also have regular shapes such as hexagonal columns and plates, bullet rosettes, dendritic crystals, etc. (Heymsfield et al., 2002; Baran, 2009; O'Shea et al., 2016). Large ice particles are generally aggregates, found both in developed cirrus (Um and McFarquhar, 2009; Baran, 2009; O'Shea et al., 2016) and as snow (Garrett et al., 2012), possibly affected by riming (freezing of supercooled liquid droplets upon contact). Schmitt and Heymsfield (2014) found that cirrus ice particles larger than about $200 \mu \mathrm{m}$ could in general be classified as aggregates.

In order to calculate the single scattering properties of ice particles, multiple methods exist. A key parameter to consider is the size parameter $x$, which relates the size of the particle to the wavelength $\lambda$ of the radiation. It is here 
defined as

$$
x=\frac{\pi D_{v e q}}{\lambda}
$$

where $D_{v e q}$ is the particle volume equivalent diameter, i.e. the diameter of a sphere with equal volume. In the Rayleigh regime, where $x \ll 1$, the shape dependence is less important, and scattering is approximately proportional to $D_{v e q}^{6}$. This is in general valid at large microwave wavelengths, such as those utilised by ground based radars, S-band (10-11 cm) for instance (Matrosov, 2007).

At higher frequencies, more sophisticated theory has to be employed. Since the main problem is the high computational demands of general scattering calculations, it has been considered desirable to approximate the particles by spheres or spheroids, where the Mie theory (Mie, 1908) and the efficient T-matrix method (Mishchenko et al., 1996), respectively, can be used. Particles that are "fluffy" have also been modelled as spheroids consisting of homogeneous ice and air mixtures. However, while such a representation can be tuned to yield reasonable results at one frequency, inconsistencies tend to appear over the spectrum, i.e. it is not a sufficient method for multi-frequency observations (Petty and Huang, 2010; Kulie et al., 2010; Geer and Baordo, 2014; Eriksson et al., 2015).

In response to this issue, there has been a trend in utilising more complex particle models and sophisticated single scattering calculation methods. Several efforts have been made to create databases containing scattering properties of ice hydrometeors (e.g. Liu, 2008; Hong et al., 2009; Kuo et al., 2016; Lu et al., 2016; Ding et al., 2017). These databases were mainly produced using the Discrete Dipole Approximation (DDA), where the particle is represented by a grid of electric dipoles (Draine and Flatau, 1994). While the method is computationally demanding, it is capable of treating arbitrary shapes.

The remaining issue is then to select appropriate particle models. Particles with intermediate size parameters $(x \approx 1)$ offer the highest modelling challenge, since the sensitivity to shape peaks in this region (Kim, 2006). As mentioned above, snow and in part cirrus clouds generally consist of aggregates. However, aggregates are generally not well covered in aforementioned databases, at least not in the ones extending into the sub-millimetre region. The "ARTS database" (Eriksson et al., 2018), which this study is related to, is a recent effort to address this problem. It is a microwave and sub-millimetre single scattering database, covering frequencies between 1 and $874 \mathrm{GHz}$, and a wide selection of ice crystals and aggregates.

Regarding radar remote sensing of snow, accurate information on the overall particle shape, and conversely ice water content (IWC), is difficult to obtain with single frequency measurements (Matrosov, 2007; Kulie et al., 2014). Collocated multi-frequency measurements are therefore desirable. Several studies (e.g. Kneifel et al., 2011; Leinonen et al., 2012; Tyynelä and Chandrasekar, 2014) have investigated radar triple-frequency signatures of particle aggregates, and shown clear differences between dif- ferent particle models (spheroid approximations, aggregates, bullet rosettes, etc.). Furthermore, such results have been used to make conclusions on microphysical parameters of observed snowfall or clouds (Kulie et al., 2014; Stein et al., 2015; Yin et al., 2017). In contrast, Leinonen and Moisseev (2015) found no large differences in triplefrequency signatures when comparing aggregates generated from different types of constituent crystals (needles, rosettes, dendrites, etc.), while the size of the crystals did have a clear impact. The impact of different amounts of riming was investigated in Leinonen and Szyrmer (2015), demonstrating a clear shift in triple frequency signatures as riming was successively applied in the model. Kneifel et al. (2015) obtained similar results by combined ground triple-frequency radar and in-situ observations, demonstrating clear differences in observed signatures between low density (fluffy) aggregates and high density (rimed) particles.

These and other studies have focused on radar applications (backscattering below $100 \mathrm{GHz}$ ). On the other hand, passive microwave and sub-millimetre observations, where extinction is of more importance, have not been considered to the same extent. Kulie et al. (2010) and Geer and Baordo (2014) looked at the impact of different particle models on simulated microwave brightness temperatures. The aggregate and snow particles in those studies were fairly simplistic however. Overall, there is a clear lack of studies focusing on the more detailed features of aggregates such as size and aspect ratio of the constituent crystals, or the overall aspect ratio of the aggregate.

This study aims to improve our understanding of relationships between aggregate parameters and single scattering properties at microwave frequencies, including submillimetre frequencies. Aggregates with a variety of properties were generated by simulation, using aggregation software developed for the ARTS database. The scattering properties of the aggregates were calculated and analysed to reveal the impact of various aggregate parameters. Mainly back-scattering $(13.4,35.6$ and $94.1 \mathrm{GHz})$ and extinction $(183.31,325.15$ and $664.00 \mathrm{GHz})$ are considered, in terms of both single particle scattering and bulk scattering. Optimally, findings here will serve as a guidance for the treatment of aggregates in future scattering databases.

\section{Method and data}

The generation of single scattering properties can be divided into two steps: 1) generating shape data, and 2) calculating the scattering properties. The method chosen in step 2 is the Discrete Dipole Approximation (DDA), specifically the software implementation called Amsterdam DDA (ADDA) by Yurkin and Hoekstra (2011).

\subsection{Particle shape data}

Shape data describe the particle shape and can be directly used as input to the scattering calculations. ADDA, 
Table 1: Overview of external shape data used. The $a$ and $b$ coefficients relate to Eq. (2). ID is the habit identifier assigned in the ARTS database (Eriksson et al., 2018).

\begin{tabular}{l|lll} 
Label & ID & $a$ & $b$ \\
\hline Hong 8-column aggregate & 8 & 65.4 & 3.00 \\
Tyynelä Fern-like dendrite & 26 & 0.125 & 2.31 \\
Evans snowflake aggregate & 1 & 0.230 & 2.41
\end{tabular}

and DDA in general, requires the shape data in the form of a discrete, equidistantly-shaped grid of dipoles (see Sec. 2.3). Because in-situ measurements typically do not provide the 3D-structure of particles, complex particle models must be provided by simulation. In order to investigate how different modelling parameters influence the overall particle shape and the associated scattering properties, a large amount of aggregate shape data have been generated. The main parameter varied is the axial ratio and size of the aggregate building blocks. For the purpose of comparison, this study also makes use of external shape data.

In order to characterise the particles, two sets of parameters are adopted here. First, the mass as a function of particle size for a given habit (i.e. a collection of differently sized particles with a common morphology) can be described by the coefficients $a$ and $b$ in the mass-size relationship:

$$
m=a D_{\max }^{b},
$$

where $D_{\max }$ is the maximum diameter. $D_{\max }$ is here defined as the diameter of the minimum circumscribed sphere. Secondly, the effective density $\rho_{\text {eff }}$ of a particle is here defined as

$$
\rho_{\mathrm{eff}}=\frac{6 m}{\pi D_{\max }^{3}}
$$

i.e. the mass divided by the minimum circumsphere volume.

\subsubsection{External shape data}

External shape data refer to shape data collected from other studies. The external shape data included here (Hong et al., 2009; Evans et al., 2012; Tyynelä et al., 2014), are summarised in Tab. 1 . The aggregate by Hong et al. (2009) is characterised by having $b=3.0$, resulting in a fixed effective density for all sizes. This stems from the fact that the model consist of a constant shape, scaled to obtain different particle sizes. That is not a realistic representation of aggregation, at least when collision is the dominant aggregation mechanism. The two remaining sources model the aggregation in an explicit manner, resulting in randomly shaped aggregates with $b$-values around 2.35 .

The scattering properties for the shape data in Tab. 1 were calculated by us, using DDA as described in section 2.3, and are a part of the ARTS database (Eriksson et al., 2018). This ensures that frequencies and temperatures not covered by the original sources are included, and any uncertainties or biases related to scattering calculations are removed. It should also be noted that the aggregates used in Tyynelä and Chandrasekar (2014) are not the same as those used for this study, but were re-generated on a dipole grid with higher resolution, by courtesy of Tyynelä on our request. Also, the ARTS database assumes totally random orientation, which was not assumed for the scattering calculations in this study. This should not pose a significant problem, because the external aggregates have fairly low aspect ratios and are unlikely to have a strong preference in orientation. However, this fact should be kept in mind when comparing the data.

\subsubsection{The Snowflake-toolkit}

A variety of aggregate type particles were generated in a stochastic fashion using a aggregation simulation software called the snowflake-toolkit (SFTK) (Rathsman, 2016). The software includes a format for general representation of aggregates and crystals by polygon meshes, ideally suited to handle hexagonal crystals. Thus, any particle that can be represented by a mesh can be used as building blocks. This study is limited to aggregates of hexagonal plates and columns, to reduce complexity in terms of analysis and computation.

The semi-physical model used for the aggregation simulation could be considered to describe a section of a cloud with a set of ice particles populating it, and is based on the model presented by Maruyama et al. (2005). At each iteration a number of events can occur: 1) growth of a new crystal, 2) collision and aggregation of two particles, 3) sedimentation out of the cloud by one particle, or 4) melting of a particle. Collision likelihood of particles $k$ and $l$ is determined by the fall-speed difference $\left|v_{k}-v_{l}\right|$ and the overlap cross-section area $\sigma_{k l}$, proportional to $\sigma_{k l}\left|v_{k}-v_{l}\right|$. The fall speed is modelled by the terminal velocity:

$$
v=\frac{\nu R_{e}}{2 \rho_{a}} \sqrt{\frac{\pi}{A}},
$$

where $\rho_{a}$ is the ambient density, $A$ the area of the particle, and $R_{e}$ the Reynolds number. The particle area $A$ is calculated in a simple manner to speed up the simulation, as the square of the maximum distance from the particle centre. Furthermore, the likelihood of melting is proportional to the inverse of the particle volume $V_{k}$, i.e. $1 / V_{k}$, while the likelihood of sedimentation is proportional to $v_{k}$. Sedimented particles are kept in memory, but do not participate in the aggregation any more. The relative importance of each event was adjusted and fine-tuned in order to achieve desirable aggregation conditions (partially from a computational point of view). In this work, the importance of melting and sedimentation has been set very low, in order to promote the growth of large aggregates.

The aggregation occurs at random orientation, however connection is only allowed between two crystal faces, such that the involved surface normals are parallel. Furthermore, the aggregation event is discarded if it leads to over- 
lap between constituent crystals, in which case the aggregation is discarded and a number (set by the user) of retries are performed until the event is completely discarded. The model includes the feature that aggregation of aggregates is possible. Fig. 1 shows examples of aggregates with crystal axis ratios $r_{\text {cryst }}=15,1$ and $1 / 15$, i.e. a column, block and plate aggregate, respectively. $r_{\text {cryst }}$ is here defined as the height $h$ of the hexagonal divided by the side base length $a$ times two, i.e. $r_{\text {cryst }}=h /(2 a)$. Each simulation results in a large particle number, up to 5000 depending on available computer memory.

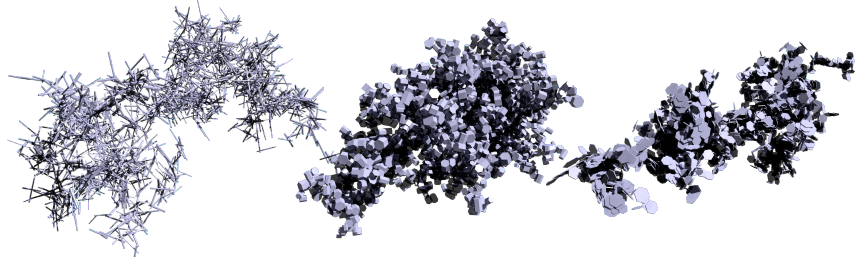

Figure 1: Example particles generated using the snowflake tool-kit, with different values of crystal axis ratio $r_{\text {cryst }}$. (Left) Column aggregate, $r_{\text {cryst }}=15$. (Middle) Block aggregate, $r_{\text {cryst }}=1$. (Right) Plate aggregate, $r_{\text {cryst }}=1 / 15$.

The DDA grids are created post-simulation, by sampling the polygon meshes used to represent the particles. The shape is represented exactly, hence dipole grids of arbitrary resolution can be created. Computation time can thus be saved by using coarser grids at lower frequencies. Each particle was sampled onto two grids with different resolutions, with dipole spacings of roughly $20 \mu \mathrm{m}$ and $70 \mu \mathrm{m}$. According to the standard dipole criterion (see Eq. (8)), allowed frequencies are then up to 670 and $190 \mathrm{GHz}$, respectively. In order to conserve the particle mass after discretisation, the dipole spacing is slightly rescaled. This results in a slight shift in particle size or $D_{\max }$.

Due to the large number of particles generated, scattering properties cannot be calculated for all the particles; a sub-set must be selected. Bulk scattering properties require that particles over a size range are considered. Selecting a sub-set as representative as possible and not impose artificial restrictions is unfortunately not straightforward. The selection used for this work is aimed at finding particles that represent the mass-size relationship in Eq. (2) as closely as possible. Fig. 2 illustrates this selection process. First, a line is fitted to the data in the logspace, then the data are divided into 10 bins in $\log \left(D_{\max }\right)$. Particles are then selected successively from each bin until a total of 30 particles have been selected. The particles selected are the ones that have the smallest $\Delta m_{i}$-values:

$$
\Delta m_{i}=\frac{\left|m_{i}-a D_{\max }^{b}\right|}{a D_{\max }^{b}},
$$

i.e. the particles that deviate the least in mass from the fitted line $\left(m_{i}\right.$ is the mass of particle $\left.i\right)$. As seen in the plot, three particles are selected from each bin, except from the eighth one, where only two particles are available. To com-

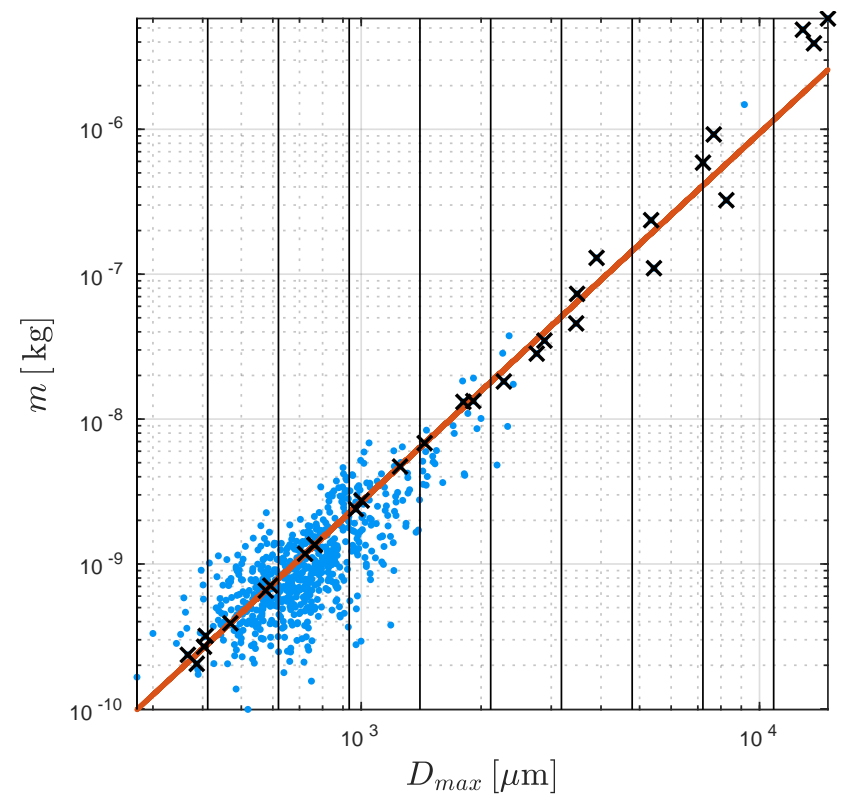

Figure 2: Illustration of the particle selection, with particle mass as a function of maximum diameter $D_{\max }$ in logarithmic scaling. Blue dots represent output from the aggregation simulation, the red line a least-square fit of Eq. (2) to the data, and the crosses are the particles selected. The black lines are the borders of the bins. In this example, three particles are selected from each bin, if available.

pensate, one additional particle is automatically selected from the first bin.

\subsection{Particle orientation}

The scattering calculations performed within this study assume horizontally random orientation of the particles. Canting or flutter angles are typically found to be approximately $10^{\circ}$ (Garrett et al., 2015; Klett, 1995), depending on the amount of turbulence. A more realistic assumption would thus be a distribution of canting angles. However, purely horizontal orientation is computationally less expensive and the goal is to investigate general features, not produce operational data. As mentioned in Sec. 2.1, the external shape data instead assume totally random orientation.

The incident light assumes a zenith incidence angle. The horizontally aligned particle is then allowed to rotate randomly around the zenith axis (z-axis), with a fixed tilt angle $\beta=0$. Mathematically, the angle distribution can be described by:

$$
p(\alpha, \beta, \gamma)=\frac{\delta(\beta) \delta(\gamma)}{2 \pi},
$$

where $\delta$ is the Dirac delta function, and $\alpha, \beta$ and $\gamma$ are the Euler angles using zyz-notation, which describes the orientation of the particle (see the ADDA manual for an illustration). The reference orientation of the particle (i.e. $\alpha=\beta=\gamma=0$ ), is determined by the principal moment of inertia axes, aligning the axis with highest associated 
inertia along the $\mathrm{z}$-axis. The axis with the smallest inertia is aligned along the $\mathrm{x}$-axis.

Apart from $\rho_{\text {eff }}$, two parameters that consider the particle orientation will be used later on. Firstly, the aggregate aspect ratio $r_{\text {agg }}$ is defined assuming a reference alignment $(\alpha=\beta=\gamma=0)$ of the particle, as the ratio of the particle span along the $\mathrm{z}$-axis to the largest particle span found in the xy-plane. Span here refers to the difference between the maximum and minimum particle coordinate along the given axis, i.e. in case of the span along z-axis it is set to $z_{\max }-z_{\min }$. Secondly, the aerodynamic area ratio $A_{\mathrm{r}}$ is defined as the ratio of the aerodynamic area to the crosssectional area of a volume equivalent sphere:

$$
A_{\mathrm{r}}=\frac{4 A_{\mathrm{aer}}}{\pi D_{v e q}^{2}} .
$$

The aerodynamic area $A_{\text {aer }}$ is set as the projected area of the particle when viewing along the $\mathrm{z}$-axis, assuming the particle is in its reference orientation. $A_{\mathrm{r}}$ is essentially a measure of how "spread out" the falling particle is in the horizontal plane, compared to a volume equivalent sphere that has $A_{\mathrm{r}}=1$. Increasingly high $A_{\mathrm{r}}$ is attained for fluffy or high aspect ratio particles.

\subsection{Calculating the scattering properties}

As explained above, the particles are represented by a discrete grid of dipoles. This grid must be fine relative to the radiation wavelength in order to ensure accurate results. This is ensured by fulfilling the standard dipole spacing criterion, i.e.

$$
\frac{\pi|m| d}{\lambda}<0.25
$$

where $m$ is the refractive index and $d$ the dipole lattice spacing (Hong, 2007). As a consequence of this criterion, the number of dipoles per wavelength (DPL) should be $\approx 22$ or higher, considering the microwave refractive index of ice. Furthermore, a minimum of 1000 dipoles is enforced at all times, ensuring that the particle shape is always discernible.

The outputs used from ADDA are the extinction crosssection $\sigma_{e}$ and the Mueller matrix $\mathbf{M}$, a 4 by 4 matrix which describes the angular dependence of the scattered light using Stokes vector notation. Its angular dependence is defined in the scattering reference frame, and is normalised such that

$$
\sigma_{s}=\frac{1}{k^{2}} \int_{4 \pi} \mathbf{M}_{11}(\hat{\mathbf{n}}) \mathrm{d} \hat{\mathbf{n}},
$$

where $\sigma_{s}$ is the scattering cross-section, $k$ the wavenumber, and $\hat{\mathbf{n}}$ the propagation vector of the scattered light.

Due to the symmetry obtained in the horizontal plane, only two DDA calculations are needed per case (i.e. combination of particle, frequency, and temperature), one each for $\mathrm{H}$-and $\mathrm{V}$-polarisation. The orientation averaged properties are retrieved analytically by rotating the Mueller matrix $360^{\circ}$ around the $\mathrm{z}$-axis (equivalent to rotation of the polarisation basis), and integrating over said rotation. This is handled internally by ADDA's internal orientation averaging routine.

\subsection{Single scattering properties}

The single scattering properties considered in this work are mainly extinction and back-scattering cross-sections. Asymmetry parameter is considered to some degree as well. For radar, three different frequency bands are considered: $\mathrm{K}_{\mathrm{u}}, \mathrm{K}_{\mathrm{a}}$ and $\mathrm{W}$, at frequencies 13.4, 35.6 and $94.1 \mathrm{GHz}$, respectively. Frequencies considered for passive microwave radiometry are $183.31,325.15$ and $664.00 \mathrm{GHz}$ (2 sub-millimetre frequencies). Refractive index is calculated using the model by Mätzler (2006). While the imaginary part of the refractive index has a significant temperature dependence (Eriksson et al., 2015), it mainly affects the absorption. Since the particles investigated in this study are fairly large, the absorption component is relatively small. Hence, only a single temperature $230 \mathrm{~K}$ is assumed. For passive microwave radiometry, extinction is of high importance, being the combined total absorption and scattering, i.e.

$$
\sigma_{e}=\sigma_{a b s}+\sigma_{s c a}
$$

Note that at large size parameters, scattering is the dominating term. Back-scattering, the most important quantity for radar applications, is extracted from the $(1,1)$ element of the Mueller matrix in the backward direction. Because the Mueller matrix is defined in the scattering reference frame, the back-scattering cross-section is

$$
\sigma_{b}=\frac{1}{k^{2}} \mathbf{M}_{11}\left(\theta=180^{\circ}\right)
$$

where $\theta$ is the scattering angle.

The corresponding efficiency $Q$ of any cross section $\sigma$ is here defined as the ratio of $\sigma$ to the cross-section area of a volume equivalent sphere, i.e.

$$
Q=\frac{4 \sigma}{\pi D_{v e q}^{2}}
$$

Asymmetry parameter $g$ is a measure of the relative amount of radiation scattered in the forward to the backward direction, important for passive microwave remote sensing (Eriksson et al., 2015). It is defined by

$$
g=\frac{2 \pi}{k^{2} \sigma_{s}} \int_{0}^{\pi} \mathbf{M}_{11}(\theta) \cos (\theta) \sin (\theta) \mathrm{d} \theta,
$$

where $\theta$ is the zenith scattering angle (there is no azimuthal dependence for horizontally aligned particles at zenith incidence angle) (Mishchenko et al., 2002). $g$ ranges from -1 to 1 , with isotropic scattering resulting in $g=0$ and $g>0$ signifying dominance of forward scattering. 
Table 2: Overview of the 11 simulation runs performed, where $\overline{r_{\text {cryst }}}$ is the nominal crystal axis ratio of given simulation. $N$ refers to the total number of particles produced from the given simulation, while $N_{a g g}$ is the number of aggregate, here defined as particles of at least 3 constituent crystals. $a$ and $b$ coefficients relate to Eq. (2) and are calculated by a least-square fit of the data, only including aggregates (i.e. no single or dual crystal particles).

\begin{tabular}{l|rrrr}
\hline$r_{\text {cryst }}$ & $N$ & $N_{\text {agg }}$ & $a$ & $b$ \\
\hline $1 / 15$ & 1233 & 570 & 0.17 & 2.35 \\
$1 / 12$ & 1378 & 525 & 0.18 & 2.34 \\
$1 / 9$ & 1387 & 514 & 0.22 & 2.35 \\
$1 / 6$ & 1433 & 488 & 0.26 & 2.34 \\
$1 / 3$ & 1374 & 415 & 0.41 & 2.38 \\
1 & 1327 & 375 & 0.57 & 2.41 \\
3 & 1259 & 373 & 0.40 & 2.43 \\
6 & 1030 & 277 & 0.33 & 2.51 \\
9 & 903 & 226 & 0.38 & 2.61 \\
12 & 718 & 170 & 0.57 & 2.74 \\
15 & 485 & 98 & 0.11 & 2.54
\end{tabular}

\subsection{Bulk scattering properties}

In practice, remote sensing measurements are always related to bulk quantities, i.e. quantities integrated over a distribution of particles. For the analysis of bulk scattering properties, an assumed particle size distribution (PSD) $N$ is required. Here we make use of the exponential distribution throughout:

$$
N\left(D_{v e q}\right)=N_{0} e^{-\Lambda D_{v e q}}
$$

where $N_{0}\left[\mathrm{~m}^{-4}\right]$ is the intercept parameter and $\Lambda[\mathrm{m}]$ the slope parameter. With $N_{0}$ constant, a decreasing $\Lambda$ results in an increased total number density and more large particles. The choice of PSD is mainly motivated by its frequency of use (Kneifel et al., 2011; Tyynelä and Chandrasekar, 2014; Leinonen and Szyrmer, 2015). Aforementioned publications make use of $D_{\max }$ in Eq. (14), however such an assumption means that a dependence on shape is included in the PSD, hence it is difficult to isolate the impact of shape on the given scattering quantity. Thus, $D_{v e q}$ is used instead, meaning that bulk quantities of different particle sets will contain the same mass content for given $N_{0}$ and $\Lambda$. $\Lambda$ is varied between $10^{3}$ to $10^{5} \mathrm{~cm}^{-1}$ and $N_{0}$ is fixed to $2.317 \cdot 10^{6} \mathrm{~m}$, following Petty and Huang (2011) based on the PSD from Sekhon and Srivastava (1970). While a fixed $N_{0}$ is not an realistic assumption, as strong correlation between $\Lambda$ and $N_{0}$ is expected, it is a common choice for this type of study (Kneifel et al., 2011; Tyynelä and Chandrasekar, 2014; Leinonen and Moisseev, 2015; Yin et al., 2017), and will only have an effect on Fig. 9 and the bottom panel of Fig. 10.

The ice mass of a volume element, commonly denoted as ice water content (IWC), is given by averaging over the particle mass $m$ :

$$
\mathrm{IWC}=\int_{0}^{\infty} m\left(D_{v e q}\right) N\left(D_{v e q}\right) \mathrm{d} D_{v e q} .
$$

Another quantity that will be used is the snowfall rate $R_{\text {leq }}$, interpreted as the liquid equivalent snowfall in $\mathrm{mm}$ per hour. It is defined as:

$$
R_{\text {leq }}=\int_{0}^{\infty} m\left(D_{v e q}\right) v_{\mathrm{t}}\left(D_{v e q}\right) N\left(D_{v e q}\right) \mathrm{d} D_{v e q},
$$

where $v_{\mathrm{t}}$ is the terminal velocity, calculated using the method by Heymsfield and Westbrook (2010). Bulk scattering quantities are calculated in a similar fashion. The extinction coefficient $\gamma_{e}$ is calculated by

$$
\gamma_{e}=\int_{0}^{\infty} \sigma_{e}\left(D_{v e q}\right) N\left(D_{v e q}\right) \mathrm{d} D_{v e q} .
$$

Similarly, the bulk Mueller matrix $\mathbf{M}_{\mathrm{b}}$ is calculated as

$$
\mathbf{M}_{\mathrm{b}}=\int_{0}^{\infty} \mathbf{M}\left(D_{v e q}\right) N\left(D_{v e q}\right) \mathrm{d} D_{v e q}
$$

Bulk back-scattering $\gamma_{b}$ can then be calculated using Eq. (11) with $\mathbf{M}_{\mathrm{b}}$ as input.

Analysis of "triple frequencies" will be performed below, and are defined here. Firstly, the effective reflectivity factor is defined as

$$
Z_{e}(\lambda)=\frac{\lambda^{4}}{\pi^{5}|K|^{2}} \int_{0}^{\infty} \sigma_{b}\left(D_{v e q}, \lambda\right) N\left(D_{v e q}\right) \mathrm{d} D_{v e q}
$$

where $K=\left(m_{w}^{2}-1\right) /\left(m_{w}^{2}+2\right)$ is the dielectric factor with $m_{w}$ the refractive index of liquid water at the wavelength $\lambda$ and assuming a reference temperature of $270 \mathrm{~K}$. The liquid water permittivity is calculated using the model by Ellison (2007). In turn, the dual wavelength ratio DWR of wavelengths $\lambda_{1}$ and $\lambda_{2}$ is

$$
\operatorname{DWR}_{\lambda_{1}, \lambda_{2}}=10 \log \left(\frac{Z_{e, \lambda_{1}}}{Z_{e, \lambda_{2}}}\right)
$$

The definition of $Z_{e}$ is intended to be independent of frequency in the Rayleigh regime, as indicated by the $\lambda^{4}$ term. Usually, one frequency is selected in the Rayleigh regime as a reference, and the others in the Mie regime, where shape dependence is high. The same set of radar frequencies are selected, i.e. at the $K_{u}, K_{a}$ and $W$ band. DWR values calculated are then $\mathrm{DWR}_{\mathrm{K}_{\mathrm{u}}, \mathrm{K}_{\mathrm{a}}}$ and $\mathrm{DWR}_{\mathrm{K}_{\mathrm{a}}, \mathrm{W}}$.

\section{Results}

\subsection{Aggregate data}

In this work, 11 aggregation simulation runs have been performed using hexagonals as constituent crystals, with crystal axis ratios $\left(r_{\text {cryst }}\right)$ varied from $1 / 15$ to 15 . The crystals generated during the simulations are not of constant size, because $a$ and $L$ are selected randomly from a Gamma distribution with a standard deviation of $25 \%$ a and $L$ are selected independently, hence $r_{\text {cryst }}$ is random as well. Furthermore, the mean value of $a$ and $L$ are selected such that the resulting mean crystal size is $D_{v e q}=120 \mu \mathrm{m}$ 


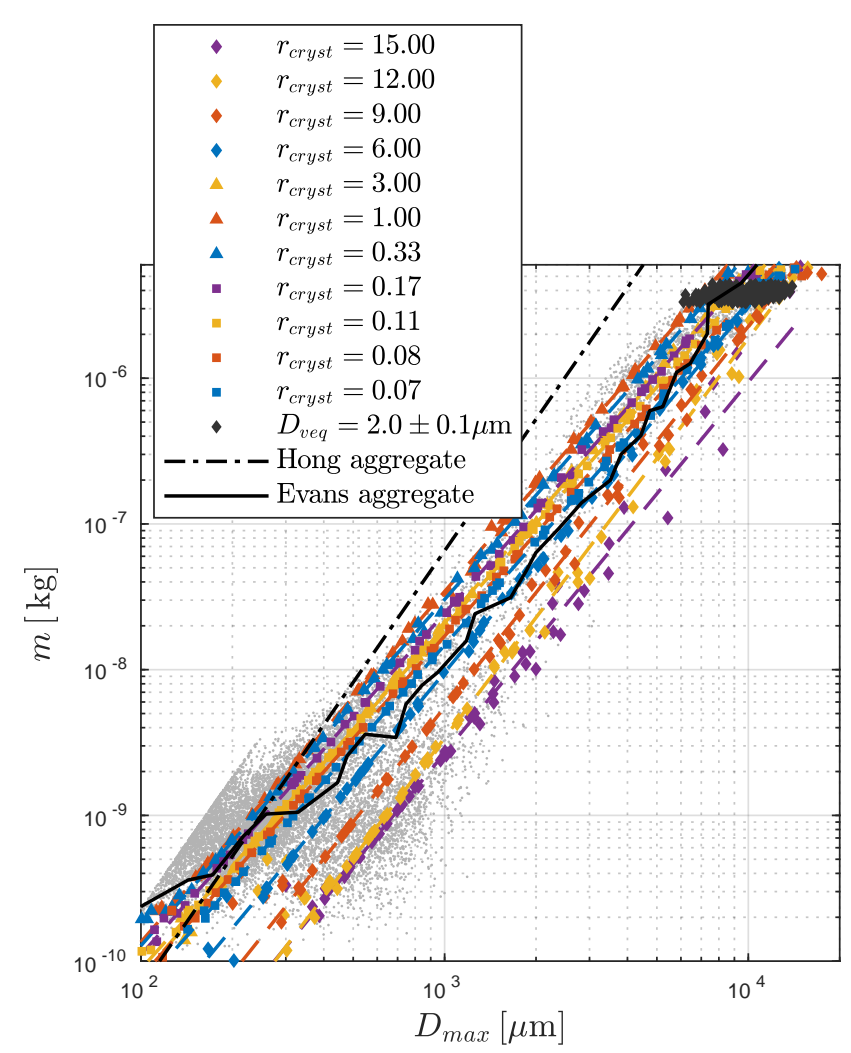

Figure 3: Particle mass as a function of maximum diameter $D_{\max }$ in logarithmic scaling for selected particles. Light grey shaded dots represent all particles from all simulations, while coloured squares and triangles are the selected particles from each simulation. The dashed lines are from Eq. (2) using the $a$-and $b$-values in Tab. 2, while the black lines represent external shape data. The Tyynelä aggregate is here omitted, in order to not clutter the plot and because it lies fairly close to the Evans aggregate. Lastly, the dark grey diamonds are particles selected close to $D_{v e q}=2$.

for all simulations. Note that $r_{\text {cryst }}>1$ implies a column type crystal.

Completely uncorrelated values of $a$ and $L$ at fixed mean values is not a fully realistic assumption, they should follow some empirical relationship (Um et al., 2015; Pruppacher and Klett, 1997) and be sampled from some probability distribution. However, we chose this simplified setup because the impact of particle shape and size upon scattering properties is easier analysed, and not obscured by assumptions on size-thickness relationship or crystal size distribution.

An overview of the simulations is available in Tab. 2 . For a particle to be considered an aggregate, at least three constituent crystals are required. The $a$ and $b$ values calculated include only these aggregates. The number of generated aggregates of high axial ratio columns are lower in general because the likelihood of successful aggregation event is relatively low, due to a zero overlap constraint.

In an attempt to pinpoint the dependence of scattering properties on different aspects of the shape data, particles were divided into three different categories. The intention is to investigate the impact of: 1) crystal axis ratio on bulk scattering, 2) crystal axis ratio on single scattering properties, and 3) crystal size on single scattering properties. In total, 1101 aggregates were selected for all categories combined. Details of each category follow in respective sub-sections below.

\subsubsection{Category 1}

Using the selection methodology described in Sec. 2.1.2, a subset of 60 particles was selected from each simulation using 20 logarithmically scaled size bins. Fig. 3 displays the selected particles' mass as a function of maximum diameter $\left(D_{\max }\right)$ and the associated mass-size relationship lines. Particle data from external sources in Tab. 1 are included as well. The particles selected using this methodology will be used to calculate and compare bulk scattering properties. This set of particles is referred to as category 1 , and contains 660 particles in total.

\subsubsection{Category 2}

For the purpose of investigating the variability of scattering properties directly, a subset of particles with a certain mass has been selected as well. They were selected from all 11 simulations as particles having $D_{v e q}=2.0 \mathrm{~mm}$, within a $\pm 10 \%$ tolerance. 234 particles were selected for this set, and they are marked as dark grey diamonds in Fig. 3. This set of particles are referred to as category 2 below. Note that 841 particles were selected in category 1 and 2, with 53 of the particles common to both categories. Also, while not displayed here, subsets of particles at $D_{v e q}=1.0 \mathrm{~mm}$ (119 particles selected) and $0.5 \mathrm{~mm}$ (97 particles selected) were created and studied as well. Analysis of these data did not reveal any significantly different features compared to the $2.0 \mathrm{~mm}$ particle data. Plots of these data, both particle properties and scattering properties, are provided as supplements.

\subsubsection{Category 3}

In the final category, denoted category 3 , the intention is to investigate the impact of constituent crystal size. Only plate aggregates of $r_{\text {cryst }}=6$ are considered, and 260 particles are selected. The particles are then scaled in size (i.e. mass), to all have a volume equivalent diameter of $D_{v e q}=2 \mathrm{~mm}$. Hence, this category consists of aggregates with a fixed mass, but with a varying number of crystals, and consequently varying crystal mass. The number of constituent crystals $N_{\text {cryst }}$ in a given aggregate ranges from 3 to 6553 . As for category 2, data were also generated for 0.5 and $1 \mathrm{~mm}$, using the same methodology and plate aggregates of $r_{\text {cryst }}=6$. These data are displayed in the supplements as well.

\subsection{Aggregate shape properties}

This section analyses the output aggregates of the snowflake-toolkit. It should be stressed that aggregation algorithms used in other studies will likely yield different 

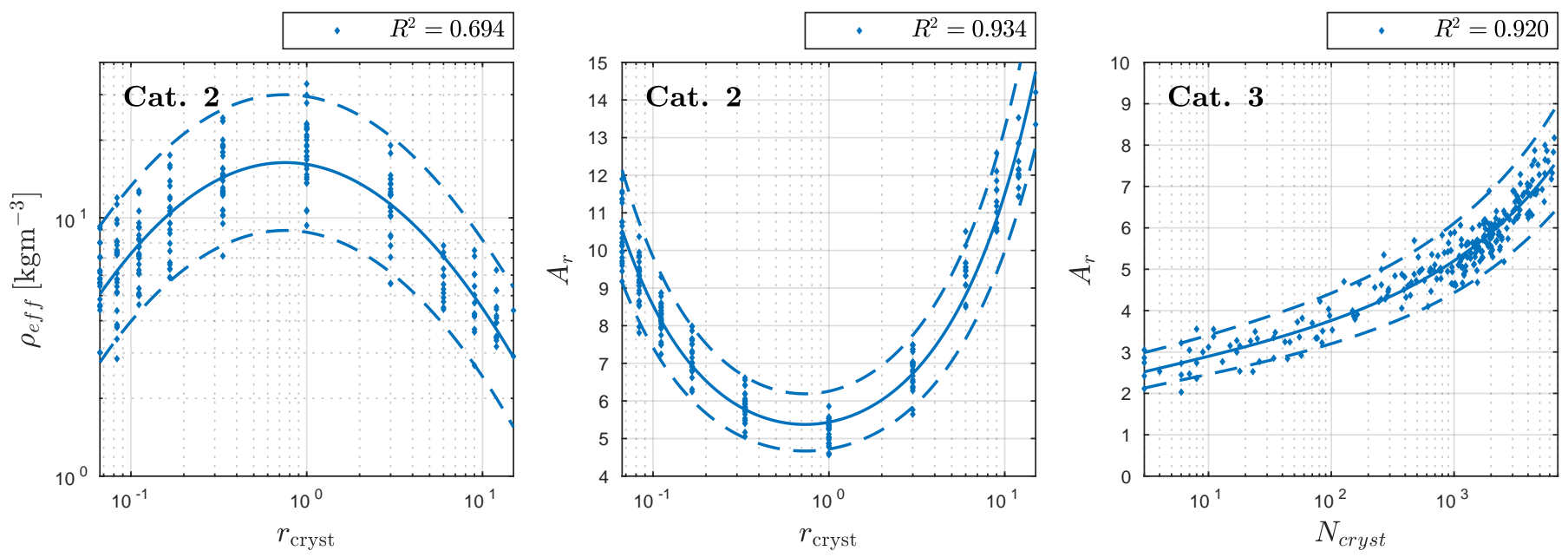

Figure 4: Aggregate parameters of category 2 and 3 shape data. (Left) Particle effective density $\rho_{\text {eff }}$ as a function of crystal axis ratio $r_{\text {cryst }}$, using category 2 data. (Middle) Aerodynamic area ratio $A_{\mathrm{r}}$ as a function of $r_{\text {cryst }}$, using category 2 data. (Right) Aerodynamic area ratio $A_{\mathrm{r}}$ as a function of the number of constituent crystals $N_{\text {cryst }}$, using category 3 data. Lines represents cubic fits.

results. Four parameters are considered: 1) $\left.r_{\text {cryst }}, 2\right)$ aggregate aspect ratio $r_{\text {agg }}$ (see Sec. 2.2), 3) effective density $\rho_{\text {eff }}$ (see Eq. (3)), and 4) aerodynamic area ratio $A_{\mathrm{r}}$ (see Eq. (7))

Fig. 4 displays a selection of resulting aggregate parameters for category 2 and 3 (other parameters are displayed in the supplements). Note that the data category used is indicated in the upper left corner of each plot. Included as solid lines are 3 -degree polynomial fits $p_{\text {fit }}$ in log-space (i.e. $\left.\log (\hat{y})=p_{\text {fit }}(\log (x))\right)$, while dashed lines represent $95 \%$ prediction bounds (i.e. with $95 \%$ probability is any new observation predicted to lie within these bounds). Also calculated is the R-squared value $R^{2}$ of each fit. Note that the fits are not attempts to find a model explaining the data. Rather, the intention is to highlight tendencies in the data and give an idea of the dependence upon a given parameter.

$\rho_{\text {eff }}$ depends on $r_{\text {cryst }}$ to a significant degree in the left panel, having fairly high $R^{2}$-values of $\approx 0.7$. Aggregates of block hexagonals $\left(r_{\text {cryst }}=1\right)$ attain the highest densities overall, while column aggregates of $r_{\text {cryst }}=15$ are generally the most fluffy. Plate aggregates are somewhat more dense than the column aggregates.

The middle panel shows $A_{\mathrm{r}}$ as a function of $r_{\text {cryst }} . R^{2}$ is higher than for $\rho_{\text {eff }}$; close to 1 . The figure indicates that block aggregates have relatively low aerodynamic areas $\left(A_{\mathrm{r}} \approx 5\right)$, while the aggregates of high axis ratio crystals have areas up to a factor 3 larger $\left(A_{\mathrm{r}} \approx 14\right)$. The correlation between $\rho_{\text {eff }}$ and $A_{\mathrm{r}}$ (not shown here, see supplements) was found to be high as well $\left(R^{2} \approx 0.8\right)$. As such, $\rho_{\text {eff }}$ and $A_{\text {r }}$ provide similar information, i.e. a measure of the overall density of particles.

$r_{\text {agg }}$ is not shown in these figures (see supplements), but was found to have little dependence upon $r_{\text {cryst }}$, with $R^{2}$ much lower than for $\rho_{\text {eff }}$ and $A_{\mathrm{r}}$ (below 0.05). $r_{\text {agg }}$ was found to lie in between 1.5 and 3 , and on average $\approx 2$.

The right panel shows $A_{\mathrm{r}}$ as a function of $N_{\text {cryst }}$, using the category 3 data. $A_{\mathrm{r}}$ increases with the $N_{\text {cryst }}$, ranging in between 2 to $8 . R^{2}$ is close to 0.9 as in the middle panel. It should also be noted that the plot indicates that $A_{\mathrm{r}}$ increases with $D_{v e q}$ in the category 1 data, at least for the aggregates of $r_{\text {cryst }}=1 / 6$ ( since $A_{\mathrm{r}}$ does not change with size scaling).

Finally, as mentioned in Sec. 3.1, data and plots were created for both category 2 and 3 at sizes of 0.5 and $1 \mathrm{~mm}$, and are provided in the supplements. These plot are highly similar in appearance as those found in Fig. 4, except for differences in the spread of the data points.

\subsection{Single scattering properties}

\subsubsection{Single scattering properties of category 1 data}

Fig. 5 displays extinction efficiency $Q_{e}$ and backscattering efficiency $Q_{b}$ as a function of $D_{v e q}$ for several frequencies. Only scattering data of category 1 are included, i.e. particles marked as coloured triangles and squares in Fig. 3. In both panels of Fig. 5, it should be noted that clear divisions can be made between scattering behaving according to Rayleigh and Mie scattering. The shift occurs roughly where the size parameter $x$ is fairly close to one. For instance, at $200 \mu \mathrm{m}$ and $325 \mathrm{GHz}, x$ is 0.7 . The exception is $13 \mathrm{GHz}$, where all sizes are within the Rayleigh regime. The back-scattering (bottom panel) follows approximately $Q_{b} \propto D_{v e q}^{4}$ at smaller sizes, consistent with Rayleigh scattering. The variance is fairly constant and similar at all frequencies in the Rayleigh regime for both extinction and back-scattering; the difference between the lowest and highest data points at a given $D_{v e q}$ is roughly a factor 2 . 

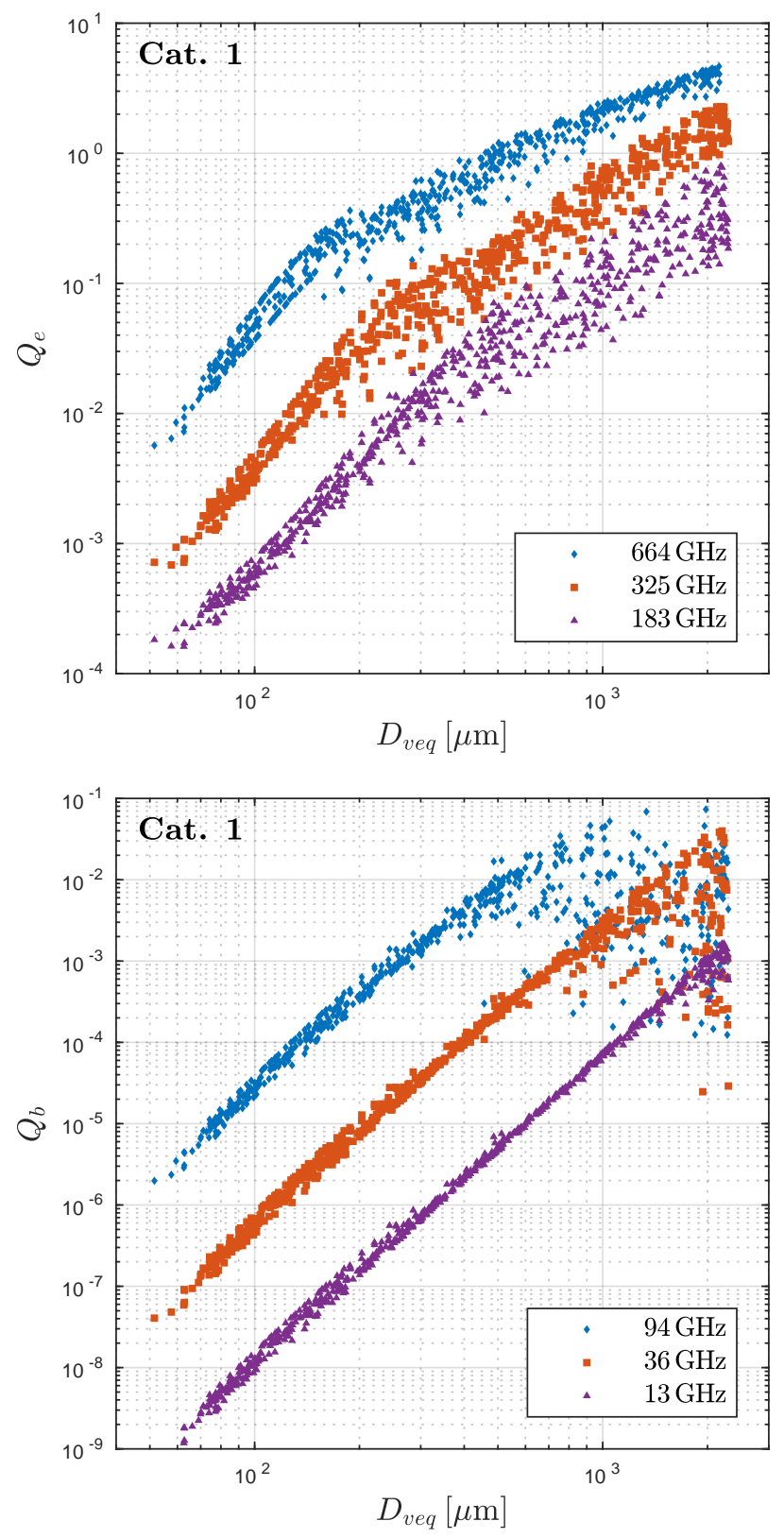

Figure 5: Extinction and back-scattering efficiencies as a function of volume equivalent diameter $D_{v e q}$. (Top) Extinction efficiency $Q_{e}$ at 183.31, 325.15 and $664.00 \mathrm{GHz}$. (Bottom) Back-scattering efficiency $Q_{b}$ at $13.4,35.65$ and $94.1 \mathrm{GHz}$.

In the Mie regime, the extinction and scattering show significantly more variance. For extinction, the spread is lowest at $664 \mathrm{GHz}$ and largest at $183 \mathrm{GHz}$ (but still inside one order of magnitude). The spread in back-scattering is much larger, up to 3 orders of magnitude at both 36 and $94 \mathrm{GHz}$.

\subsubsection{Radar back-scattering}

Fig. 6 displays back-scattering efficiency for category 2 $\left(D_{v e q}=2 \pm 0.2 \mathrm{~mm}\right)$ and $3\left(D_{v e q}=2 \mathrm{~mm}\right)$ data. Fitted lines and $R^{2}$ are calculated with the same methodologies as in Sec. 3.1. The two leftmost panels show category 2 data and the rightmost category 3 data.

Tendencies with respect to $r_{\text {cryst }}$ are observed in the left panel. $R^{2}$-values are fairly high at 13 and $36 \mathrm{GHz}$. At $36 \mathrm{GHz}, R^{2}$ is roughly equal to 0.7 , with block aggregates being associated with the highest back-scattering. The dependence on $\rho_{\text {eff }}$ and $A_{\mathrm{r}}$ (not shown here, see supplements) is similar in comparison to $r_{\text {cryst }}$. Of those three parameters, highest correlations are achieved for $r_{\text {cryst }}$ and the lowest for $\rho_{\text {eff }}$, for which $R^{2} \approx 0.4$ at $36 \mathrm{GHz}$. $A_{\mathrm{r}}$ has slightly lower $R^{2}$-values than for $r_{\text {cryst }}$. In the middle panel, the dependence upon $r_{\text {agg }}$ is even lower, with $R^{2}$ less than 0.13 at all frequencies. A small tendency towards increased $Q_{b}$ with $r_{\text {agg }}$ is seen, but the data density is somewhat lacking at $r_{\text {agg }}>2.5$, hence definite conclusions cannot be made.

The right panel of Fig. 6 shows back-scattering for category 3 data as a function of $N_{\text {cryst }}$. As a reminder, the crystal sizes are scaled such that each aggregate has the same mass (i.e. $D_{v e q}=2 \mathrm{~mm}$ ). Aggregates with only a few crystals are more efficient scatterers in comparison to aggregates consisting of a high number of crystals. This relationship is overall more compact in comparison to the panels to the left. Replacing $N_{\text {cryst }}$ with $A_{\mathrm{r}}$ on the x-axis (shown in supplements) yields somewhat lower $R^{2}$-values.

The difference between frequencies should be noted. $13.4 \mathrm{GHz}$ shows relatively low variance in all panels, with high dependence on $r_{\text {cryst }}$ and $N_{\text {cryst }}$. On the other hand, the back-scattering at 35.6 and $94.1 \mathrm{GHz}$ shows a very high spread. $94 \mathrm{GHz}$ seems to be only well explained by $N_{\text {cryst }}$ in the right panel, however the spread is very large above $N_{\text {cryst }}=1000$.

Corresponding plots of 0.5 and $1 \mathrm{~mm}$ data are found in the supplements. The impact upon back-scattering efficiency was generally found to be lower, with some exceptions. Exceptions are scattering at $94 \mathrm{GHz}$, having somewhat higher $R^{2}$-values for all parameters at both 0.5 and $1 \mathrm{~mm}$. Furthermore, $R^{2}$ is also higher for $N_{\text {cryst }}$ at all frequencies. Overall, the observations in previous paragraphs are preserved at these sizes, except that there are more combinations of frequencies and sizes in the Rayleigh regime, and that the variance is generally reduced.

\subsubsection{Extinction}

In a similar fashion to Fig. 6, extinction efficiency is shown in Fig. 7 for category 2 and 3 data. $Q_{e}$ shows a clear dependence upon $r_{\text {cryst }}$ in the left panel. $R^{2}$ is in the order of 0.9 at 183 and $325 \mathrm{GHz}$. Smaller values are found for $664 \mathrm{GHz}$, likely due to under-fitting of the polynomial fit (data points can be seen to fluctuate relative to the line). There is also a shift in the shape of the fitted curves between 325 and $664 \mathrm{GHz}$, from a negative to a positive second derivative. In the middle panel, $A_{\mathrm{r}}$ compares similarly to $r_{\text {cryst }}$, with the exception that a clear improvement is seen for $664 \mathrm{GHz}$ data. The shapes of the curves also show progression with frequency, from positive to negative second derivatives as frequency increases. 

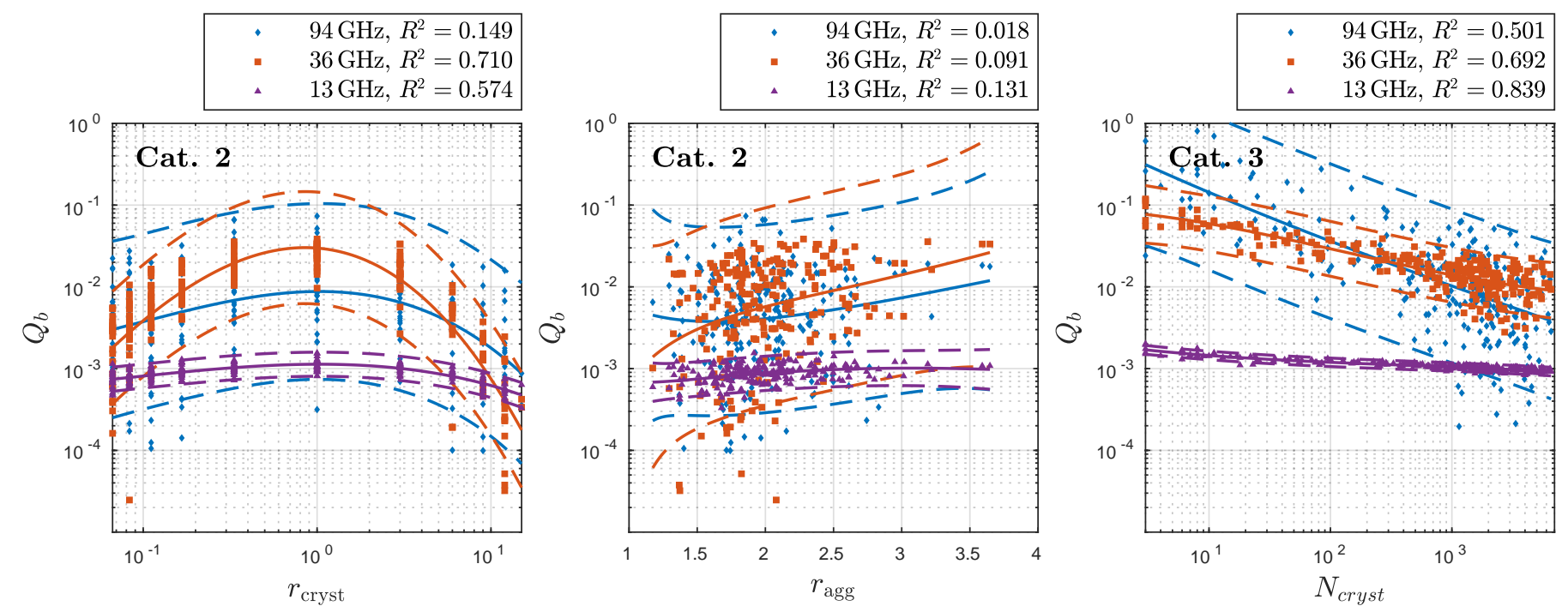

Figure 6: Back-scattering efficiencies $Q_{b}$ as a function of different parameters, at 13.4, 35.6 and $94.1 \mathrm{GHz}$. The temperature is 230 K. (Left) $Q_{b}$ as a function of crystal axis ratio $r_{c r y s t}$, using category 2 data. (Middle) $Q_{b}$ as a function of aggregate aspect ratio $r_{\text {agg }}$, using category 2 data. (Right) $Q_{b}$ as a function the crystal number $N_{\text {cryst }}$, using category 3 data.

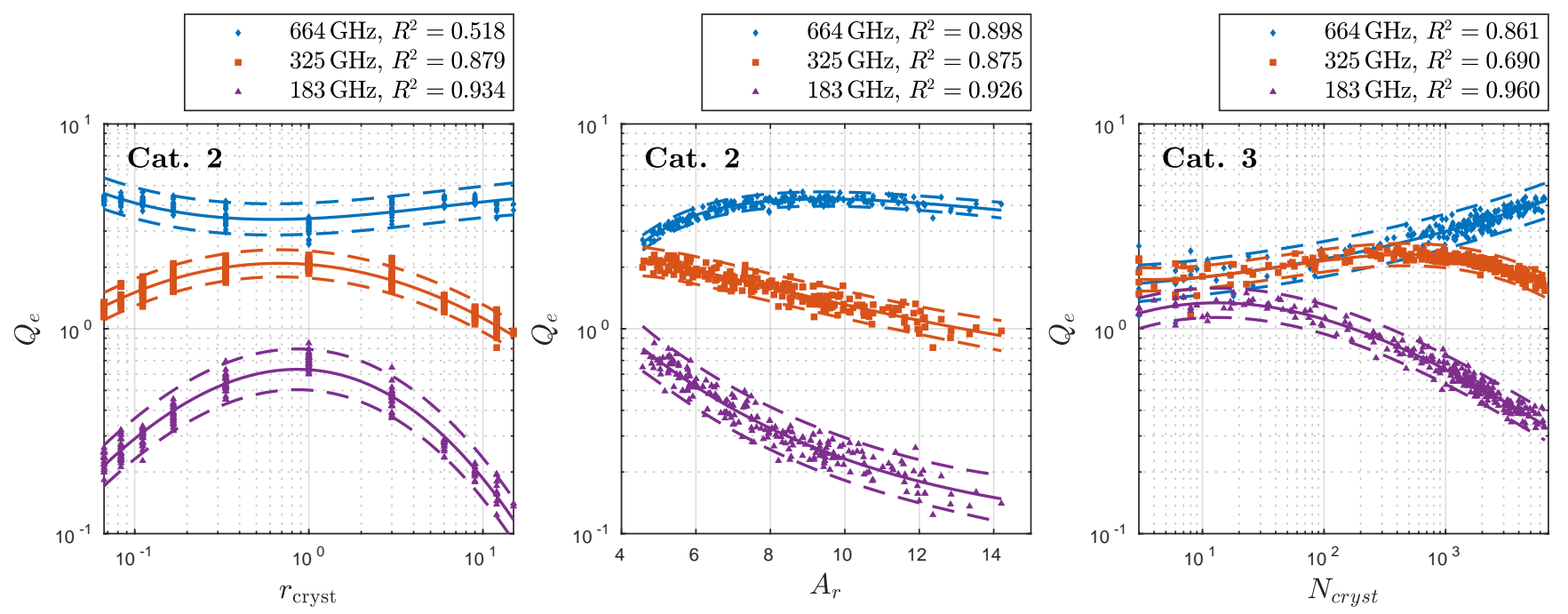

Figure 7: Extinction efficiencies $Q_{e}$ as a function of different parameters, at $183.31,325.15$ and $664.00 \mathrm{GHz}$. The temperature is $230 \mathrm{~K}$. (Left) $Q_{e}$ as a function of crystal axis ratio $r_{c r y s t}$, using category 2 data. (Middle) $Q_{e}$ as a function of aerodynamic area ratio $A_{\mathrm{r}}$, using category 2 data. (Right) $Q_{e}$ as a function of the constituent crystal number $N_{\text {cryst }}$, using category 3 data.

Using $\rho_{\text {eff }}$ as a descriptor (see supplements) yields somewhat worse results in comparison to $r_{\text {cryst }}\left(R^{2}\right.$ is 0.7 and 0.6 for 183 and $325 \mathrm{GHz}$, respectively). $664 \mathrm{GHz}$ is an exception, which sees an improvement $\left(R^{2} \approx 0.7\right)$, though still worse than for $A_{\mathrm{r}}$. Using $r_{\text {agg }}$ (see supplements), resulted in almost no dependence, $\left(R^{2} \approx 0.001\right.$ for 183 and $325 \mathrm{GHz}$, and $R^{2} \approx 0.07$ for $664 \mathrm{GHz}$ ).

The correlation to $N_{\text {cryst }}$, for category 3 data, is shown in the left panel to be high, $R^{2} \geq 0.08$ at all frequencies (close to 1 for $183 \mathrm{GHz}$ ). Once again, a shift in the appearance of the curves is seen between 325 and $664 \mathrm{GHz}$. Using $A_{\mathrm{r}}$ on the x-axis (see supplements) yields even better results, $R^{2}$ improves at all frequencies. It was also found that the data points $\left(Q_{e}\right.$ as a function of $\left.A_{\mathrm{r}}\right)$ occupy roughly the same points as in the middle panel, i.e. $A_{\mathrm{r}}$ is an equally good descriptor for category 2 and 3 data.

Once again, corresponding plots of 0.5 and $1 \mathrm{~mm}$ data are available as supplements. There are some interesting differences. For instance, the shift of curvature in the left panel between 325 and $664 \mathrm{GHz}$, is not found in the 0.5 and $1 \mathrm{~mm}$ data. Otherwise, $R^{2}$-values are with some exceptions either similar in size or lower.

\subsubsection{Asymmetry}

The asymmetry parameter $g$ as a function of $r_{\text {cryst }}$ is considered in the top panel of Fig. 8. $g$ varies in between roughly 0.7 and 0.9 , and relatively high dependence upon 

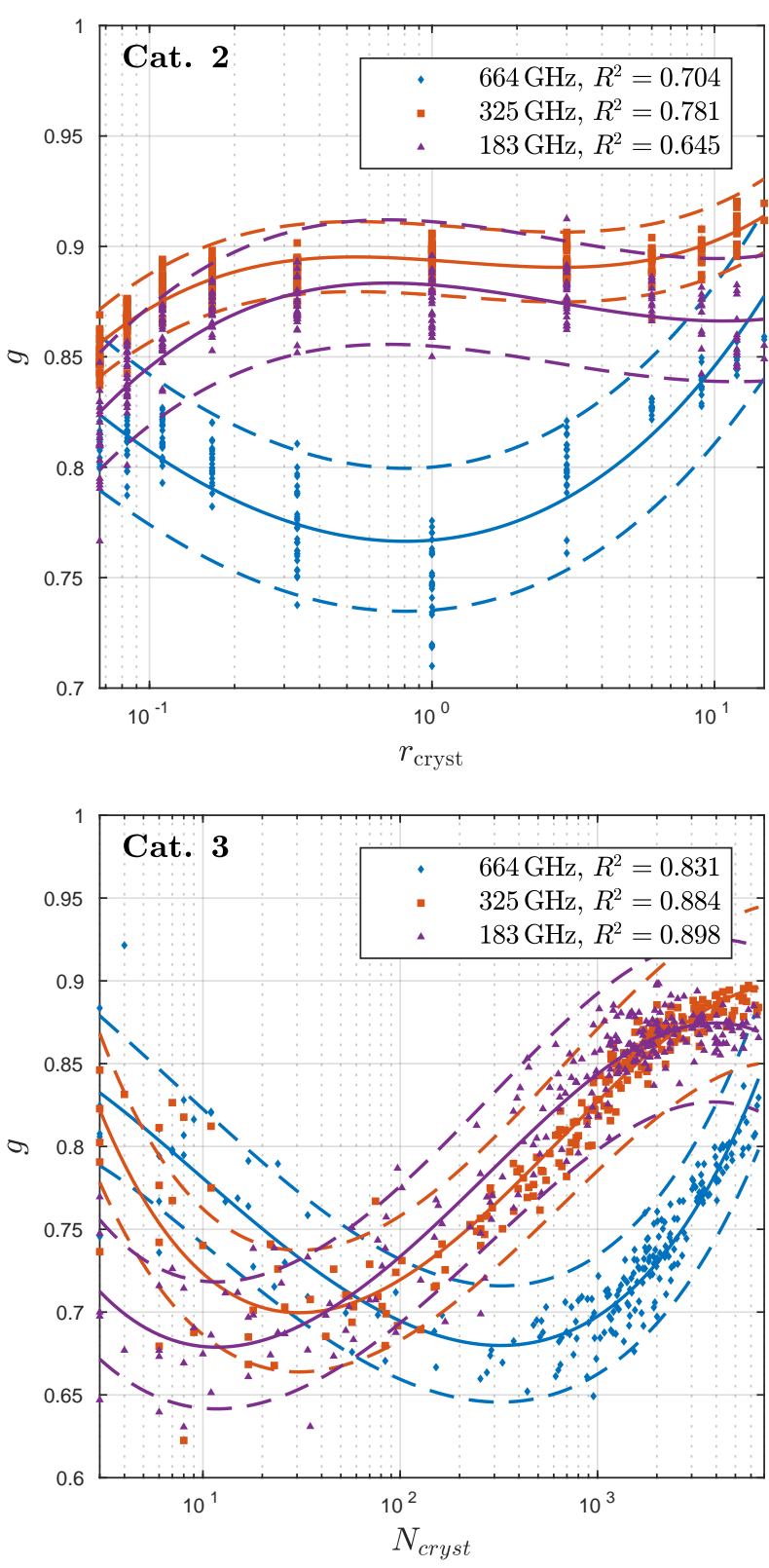

Figure 8: Asymmetry parameter $g$ as a function of different parameters, at $183.31,325.15$ and $664.00 \mathrm{GHz}$, and a temperature of 230 K. (Top) $g$ as a function of crystal axis ratio $r_{\text {cryst }}$, using category 2 data. (Bottom) $g$ as a function of the crystal number $N_{\text {cryst }}$, using category 3 data.

$r_{\text {cryst }}$ is demonstrated at all frequencies $\left(R^{2}\right.$ at least 0.65 for all frequencies). As was the case for $Q_{e}$, the fitted lines switch curvature between 325 and $664 \mathrm{GHz}$. The dependence on $\rho_{\text {eff }}$ and $A_{\mathrm{r}}$ (see supplements) is reduced at 183 and $325 \mathrm{GHz}$, but similar at $664 \mathrm{GHz}$. Negligible dependence with respect to $r_{\text {agg }}$ was found, with $R^{2}<0.05$ at all frequencies.

Finally, $g$ as a function of $N_{\text {cryst }}$ is shown in the bottom panel of Fig. 8, for category 3 data. $R^{2}$ of $N_{\text {cryst }}$ are higher than for $r_{\text {cryst }}$, with values of at least 0.83 for all frequencies. Also, for each frequency, a local minimum is found for the fitted lines, and the $N_{\text {cryst-values of these minima }}$ increase with frequency.

Similar plots for data at 0.5 and $1 \mathrm{~mm}$, are once again available in the supplements. For category 2 data, the dependence of $g$ upon $r_{\text {cryst }}$ is clearly decreasing for lower values of $D_{v e q}$, and a larger variance in $g$ is seen. At $500 \mu \mathrm{m}, g$ reaches values as low as 0.1 . The category 3 data shows similar tendencies. $R^{2}$ take on fairly similar values at $1 \mathrm{~mm}$, compared to the bottom panel of Fig. 8, but drops significantly at $0.5 \mathrm{~mm}$. The spread in $g$ increases in overall.

\subsection{Bulk scattering properties}

Bulk scattering properties are treated in this section. All figures assume an exponential PSD in $D_{v e q}$ (see Eq. (14)), and a temperature of $230 \mathrm{~K}$. Scattering data using external shape data (see Sec. 2.1.1) have been included for reference.

\subsubsection{Passive microwave and sub-millimetre extinction}

The top panel of Fig. 9 displays bulk extinction coefficient $\left(\gamma_{e}\right)$ as a function of ice water content, at 183 and $664 \mathrm{GHz}$ respectively, with data divided into lines of different $r_{\text {cryst }}$. The habits from the external sources (summarized in Tab. 1) are included as dotted lines. Totally random orientation is assumed for the external habits, as mentioned earlier. Horizontally random orientation is assumed otherwise.

At $183 \mathrm{GHz}$, aggregates of $r_{\text {cryst }} \approx 1$, (associated with high $\rho_{\text {eff }}$ in Fig. 4) are seen to have the highest $\gamma_{e}$-values. Conversely, column aggregates $\left(r_{\text {cryst }}>1\right)$ have the lowest extinction, roughly a factor 6 lower, over the whole IWC range. The plate aggregates $\left(r_{\text {cryst }}<1\right)$ occupy the space in between the block and column aggregates. Overall, increasing the aspect ratio of the crystals (i.e. deviation from $r_{\text {cryst }}=1$ ) tends to lower the extinction. The extinction at $664 \mathrm{GHz}$, shows less variance in comparison to $183 \mathrm{GHz}$, the spread is roughly a factor 2 , with a high degree of overlap between the lines.

In the bottom panel of Fig. $9, \gamma_{e}$ is shown as a function of snowfall rate $R_{\text {leq }}$ (Eq. 16). Note that $v_{\mathrm{t}}$ has an approximately inverse dependence on $A_{\mathrm{r}}$ ( $v_{\mathrm{t}}$ increases with decreasing $A_{\mathrm{r}}$ ), which according to the middle panel in Fig. 4 , is generally smaller for aggregates of $r_{\text {cryst }} \approx 1$. At both frequencies, replacing the x-axis from IWC to $R_{\text {leq }}$ results in a decrease in spread, to the point where the lines significantly overlap at $664 \mathrm{GHz}$. At $183 \mathrm{GHz}$, the spread is a factor of roughly 3 in the upper end of $R_{\text {leq }}$, and roughly 2 at the lower end. Thus, replacing IWC with $R_{\text {leq }}$ reduces the spread in $\gamma_{e}$ by a factor of roughly 2 .

Regarding the external habits, the Hong aggregate, which is composed of large solid columns, demonstrates the highest extinction of all habits at $183 \mathrm{GHz}$, in the top panel. The Evans and Tyynelä aggregates demonstrate tendencies fairly similar to the low $r_{\text {cryst }}$ aggregates, having fairly high extinction at both frequencies. The Hong 

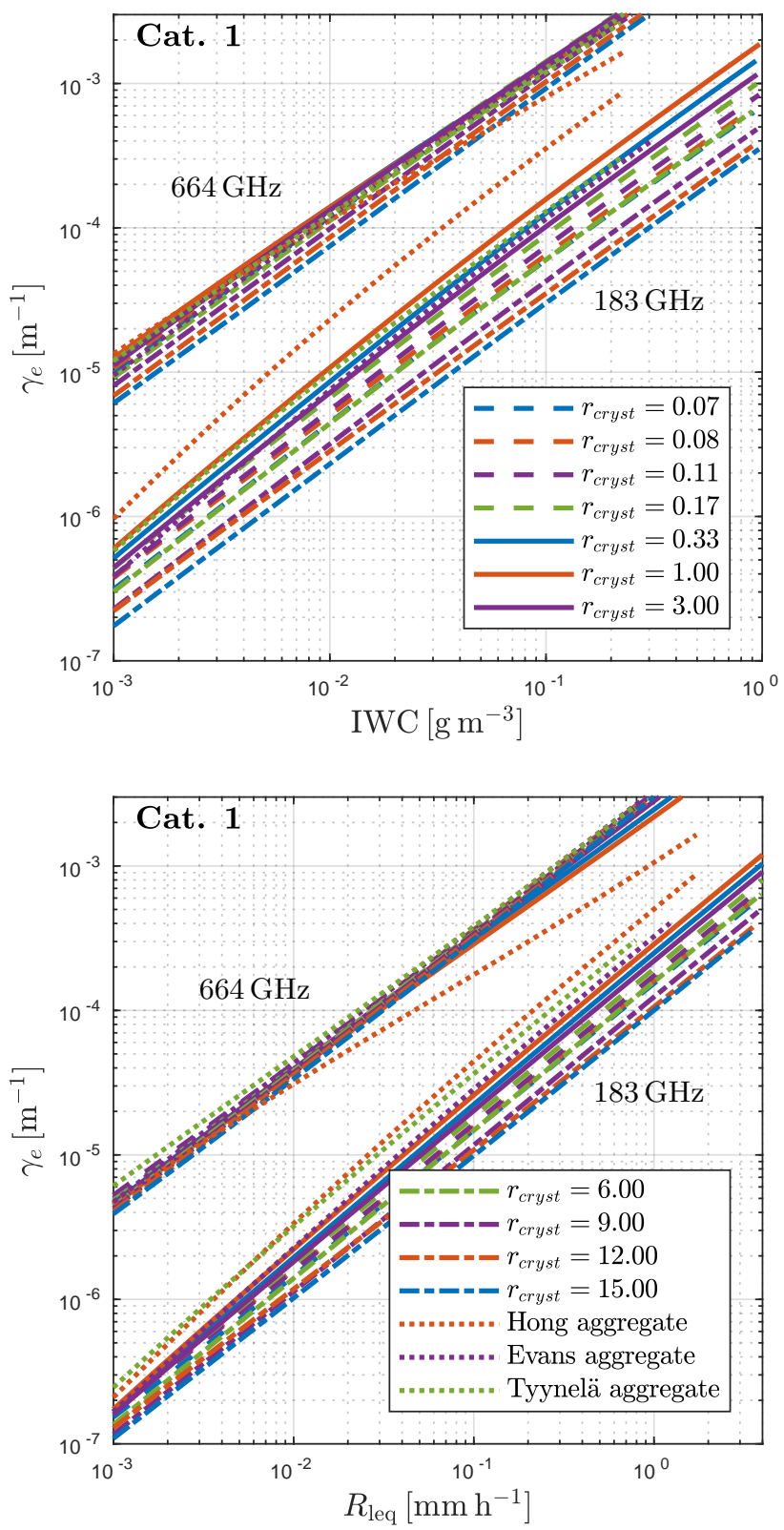

Figure 9: Bulk extinction $\gamma_{e}$ separated into lines of crystal axis ratio $r_{\text {cryst }}$ and 3 external habits. Two frequencies are shown in both panels, where the upper and bottom collection of lines correspond to 664.00 and $183.31 \mathrm{GHz}$, respectively. Temperature is $230 \mathrm{~K}$ and an exponential PSD in $D_{v e q}$ was assumed. (Top) $\gamma_{e}$ as a function of ice water content. Note that the blue dashed line is hidden behind the green dot-dashed line. (Bottom) $\gamma_{e}$ as a function of liquid equivalent snowfall $R_{\text {leq }}$, in $\mathrm{mm}$ per hour. The legends are common for the two panels.

aggregate stands out in the bottom panel at $664 \mathrm{GHz}$, having relatively low extinction at given snowfall rates.

\subsubsection{Radar back-scattering and triple frequency signa- tures}

The top panel of Fig. 10 displays triple frequency signatures. Lines are divided into $r_{\text {cryst }}$ and external particles are included as in the previous figure. Aggregates of dif-
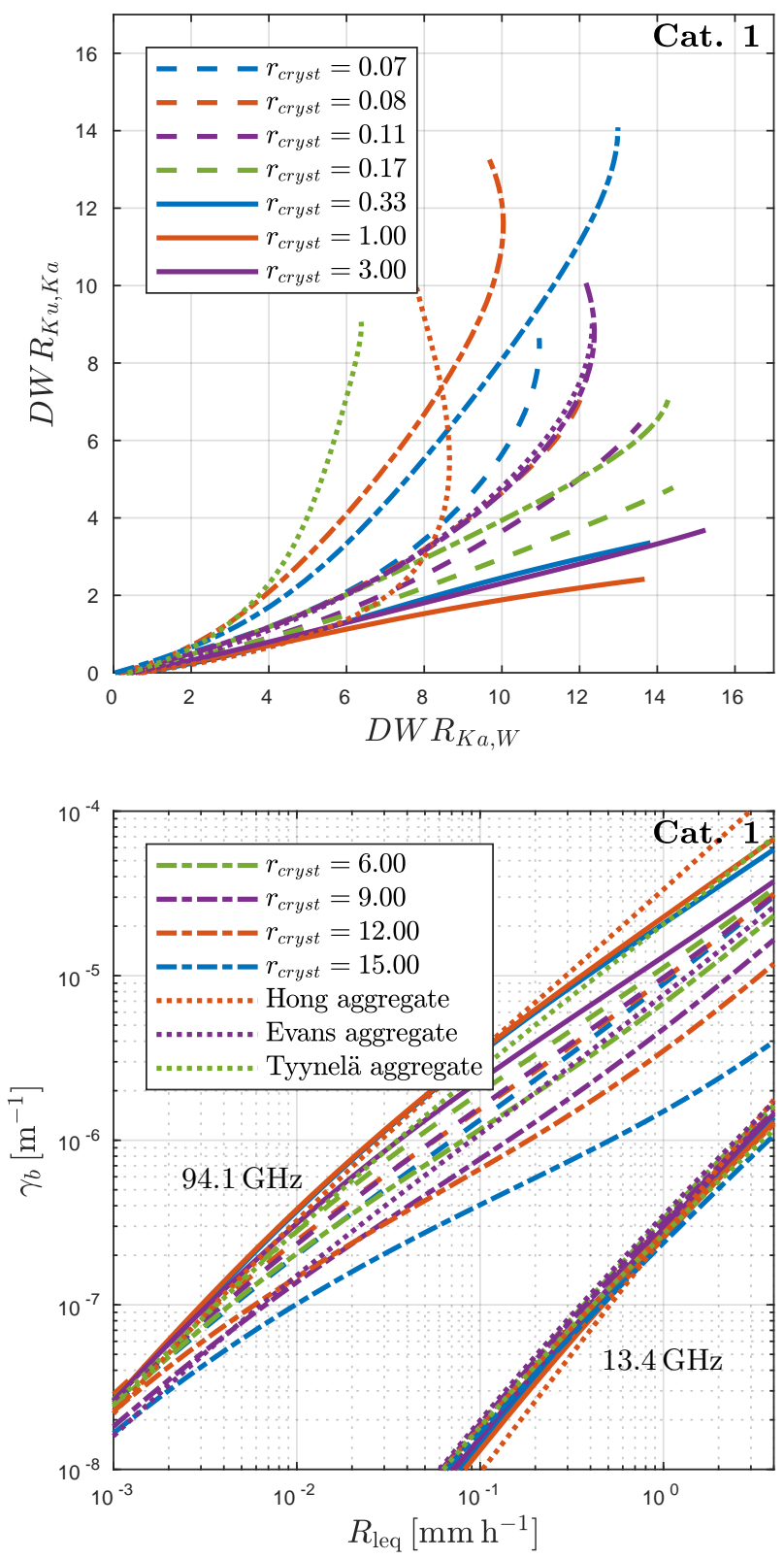

Figure 10: Triple frequency signatures and bulk back-scattering coefficients, using radar frequencies of 13.4 ( $\mathrm{K}_{\mathrm{u}}$-band), $35.6\left(\mathrm{~K}_{\mathrm{a}}\right.$-band) and $94.1 \mathrm{GHz}$ (W-band). Temperature is $230 \mathrm{~K}$ and an exponential PSD was assumed. Lines are divided into different $r_{\text {cryst-values. }}$ (Top) Triple frequency signatures. (Bottom) Bulk back-scattering coefficient $\gamma_{b}$ as a function of $R_{\text {leq }}$, at 94 and $13.4 \mathrm{GHz}$ in the upper and lower collection of lines, respectively. The legends are common for the two panels.

ferent $r_{\text {cryst }}$-values produce distinct signatures. The block aggregate $\left(r_{\text {cryst }}=1\right)$ occupies the lower right portion of the top panel. As $r_{\text {cryst }}$ deviates from 1, the lines are bent counter-clockwise. Column aggregates, $r_{\text {cryst }}=12$ in particular, are bent the furthest, while plate aggregates occupy the intermediate area.

As a test, triple frequency signatures were produced with $D_{v e q}$ replaced by $D_{\max }$ in the particle size distribu- 
tion (Eq. 14). The plot is available in the supplements. Overall, the appearance is similar to the top panel of Fig. 10. A small counter-clockwise shift of varying magnitude can be observed for all habits. For $r_{\text {cryst }}=1$ only a negligible shift is found, while for $r_{\text {cryst }}=15$ the shift is up to $2 \mathrm{~dB}$.

Bulk back-scattering coefficient $\left(\gamma_{b}\right)$ as a function of $R_{\text {leq }}$ are displayed in the bottom panel. $\gamma_{b}$ has a fairly high spread at $94.1 \mathrm{GHz}$, roughly a factor 10 difference between lowest and highest line at $R_{\text {leq }}$ higher than $1 \mathrm{~mm} \mathrm{~h}^{-1}$ (excluding the external habits). At $13.4 \mathrm{GHz}$ (lower collection of lines), the spread is much smaller at all $R_{\text {leq-values. }}$ Back-scattering at $35.6 \mathrm{GHz}$ is not displayed, in order to not clutter the plot. However, it behaves fairly similar to $\gamma_{e}$ at $94 \mathrm{GHz}$, with a slightly lower spread. Back-scattering as a function of IWC is not displayed. The spread was found to be higher in comparison, at $94 \mathrm{GHz}$ the spread is a factor 2 higher than when using $R_{\text {leq }}$. Hence, the decrease in spread at $94 \mathrm{GHz}$ between IWC and $R_{\text {leq }}$ is similar to what was found for the extinction $\gamma_{e}$. At $13.4 \mathrm{GHz}$ the difference in spread is about $50 \%$.

\section{Discussion}

Simulations by the snowflake tool-kit with different values of constituent crystal axis ratio $\left(r_{\text {cryst }}\right)$ produced aggregates of fairly distinct features. Judging by the lines in Fig. 3, some of the generated aggregates, the $r_{\text {cryst }}=6$ aggregate for instance, yield a mass-size relationship fairly similar to the Evans aggregate. Furthermore, the aggregates have values of $b$ in between 2.35 and 2.74 (Tab. 2). Measurements and simulations by Heymsfield et al. (2002) and Westbrook et al. (2004) argue for $b=2$, while Mitchell (1996) estimated $b$ to lie in between 2.1 and 2.45 for aggregates and 2.8 for lump graupel, using in-situ measurements. Also, Locatelli and Hobbs (1974) reported empirical expressions with $b$ equal to 1.9 for unrimed aggregates of plates, side planes bullets and columns, and 1.9 for unrimed aggregates of dendrites. Our aggregates could thus be considered to have fairly high $b$-values (although comparable to the aggregates included from other sources). The high $b$-values can partly be explained by the settings used for our aggregation simulations, which were required in order to generate a large enough set of aggregates with high mass. Limited test simulations revealed that aggregates of $b$-values closer to two was indeed possible.

The obtained effective density $\left(\rho_{\text {eff }}\right)$ and area ratio $\left(A_{\mathrm{r}}\right)$ showed a clear dependence upon $r_{\text {cryst }}$. As one would expect, constituent crystals of either low or high axis ratio result in less dense aggregates, while crystals of axis ratios close to one result in dense aggregates. $a$-and $b$-values derived from in-situ measurements by Mitchell (1996) give values of $\rho_{\text {eff }}$ in between 5 and $16 \mathrm{~kg} \mathrm{~m}^{-3}$ for aggregates of $D_{v e q}=2 \mathrm{~mm}$, which is covered by the data points in the left panel of Fig. 4. The number of constituent crystals $\left(N_{\text {cryst }}\right)$ in a given aggregate was also shown to have a clear impact on these parameters. An increasing $N_{\text {cryst }}$ results in particles of lower density (given that the total particle mass is fixed), and larger aerodynamic or projected area. These arguments should for now only be considered to apply to particles generated using the snowflake tool-kit, as it cannot be established whether they apply to other aggregate generating algorithms as well; we are unaware of any other studies performing similar tests with different algorithms. Regardless, the produced aggregates have $b$-values and effective densities that cover a fairly large span, and to some extent matches values observed in nature. The fluffiest aggregates, being the ones composed of columns, likely best serve as proxies for snow, while the dense block aggregates best serve as proxies for rimed aggregates. This is supported by the general appearance of the renderings in Fig. 1.

Regarding the single scattering properties, extinction efficiency $\left(Q_{e}\right)$ and back-scattering efficiency $\left(Q_{b}\right)$ were found to be clearly correlated to all of the investigated parameters, except for the aggregate aspect ratio $\left(r_{\mathrm{agg}}\right)$. $Q_{e}$ in particular showed very high dependence, regardless of whether category 2 or 3 data was used. $N_{\text {cryst }}$ was found to have high impact on both $Q_{e}$ and $Q_{b}$. $Q_{b}$ generally decreases with $N_{\text {cryst }}$, while $Q_{e}$ shows a more complex behaviour. For 183 and $325 \mathrm{GHz}$, local maxima were found at increasing values of $N_{\text {cryst }}$. Hypothetically, such a maximum could exist for $664 \mathrm{GHz}$, however, scattering data with high enough values of $N_{\text {cryst }}$ were not produced to confirm this.

$Q_{e}$ at $664 \mathrm{GHz}$ seems to behave differently compared to extinction at the other frequencies. In specific, the sign of the second derivative of the fitted lines are seen to shift between 325 and $664 \mathrm{GHz}$ for most parameters. As an example, in the middle panel of Fig. 7, extinction at $664 \mathrm{GHz}$ increases with $A_{\mathrm{r}}$-values up to 10 , while at $325 \mathrm{GHz}$ it decreases at all $A_{\mathrm{r}}$-values. This is an indication of scattering more in line with geometric optics, with the extinction being more related to the geometric cross-section (note that the size parameter $(x)$ is roughly 14 at $664 \mathrm{GHz}$ and $D_{v e q}=2 \mathrm{~mm}$ ).

$Q_{b}$ could be explained by the considered aggregate parameters as well, albeit to a lower degree. The scattering at $13.4 \mathrm{GHz}$ is characterised by Rayleigh scattering, i.e. it is mostly dependent on the mass and of low variance at given mass. The higher frequencies are in the Mie regime and demonstrate high shape dependency. $Q_{b}$ at $94 \mathrm{GHz}$ showed very high variance in particular, and could not be well explained by any parameter investigated. There could be interference effects occurring in sub-structures of the aggregates that are not well captured by any of the investigated shape parameters removed text in parenthesis.

It is somewhat surprising that $r_{\text {agg }}$ showed such low dependence on $r_{\text {cryst }}$ and marginal impact on the scattering properties. This is likely in part due to the fact that the aggregation simulations assume totally random orientation for the aggregating particles. Also, since the definition of $r_{\text {agg }}$ used here could be considered to depend on the extreme features of the particles (i.e. maximum exten- 
sion along two directions), it may be a too ambiguously defined variable. However, the same argument could be applied to the $\rho_{\text {eff }}$ (as defined here), since the size of the minimum circumsphere is determined by particle extrema as well. Yet this parameter was seen to be a fairly good descriptor of scattering properties in general. It should be noted that high aspect ratios of aggregates does not seem to be supported by observations. Garrett et al. (2015) found that the median aspect ratio for aggregates were 1.66 for aggregates, which is less than the mean aspect ratio $r_{\text {agg }}$ of roughly 2 found for the aggregates generated here. Garret's definition of aspect ratio is slightly different compared to the one used here, reflecting the fact that the aspect ratio is calculated from 2D-images and not from 3D-models.

An important comparison is that of passive and active single scattering properties. Comparing on one hand the two panels of Fig. 5, and on the other hand Fig. 6 to 7, reveals that back-scattering generally shows more shapeinduced variance than extinction, at least when considering size parameters above 1 . This suggests that passive measurements have an advantage when retrieving ice mass. The exception to this is back-scattering at $13.4 \mathrm{GHz}$, characterised by Rayleigh scattering throughout the considered particle size range, showing perhaps the smallest spread overall. Note that comparison between passive and active measurements is only relevant when both instruments have sufficient sensitivity to the retrieved quantity. Also, radar still has the advantage overall when considering vertical resolution, footprint size, etc

Continuing on the topic of passive scattering properties, it is also of interest that $664 \mathrm{GHz}$ seems to show a fairly narrow spread in extinction compared to other frequencies, both for bulk and single scattering properties. This is supported by the top panel in Fig. 5, where $Q_{e}$ at $664 \mathrm{GHz}$, shows less variance than the other frequencies. Also, in Fig. 9, the spread of the $664 \mathrm{GHz}$ lines is very small. The implication is that since extinction at $664 \mathrm{GHz}$ is the least sensitive to shape, it has an advantage among the investigated frequencies for retrieving ice mass by passive measurements. Radiation attenuated at $183 \mathrm{GHz}$, is more impacted by shape.

The asymmetry parameter $(g)$ was seen to behave differently at $664 \mathrm{GHz}$ compared to other frequencies as well (see top panel of Fig. 8). It has a minimum at $r_{\text {cryst }}=1$ and increases as $r_{\text {cryst }}$ deviates from 1 . Eriksson et al. (2015) investigated the importance of $g$ in passive microwave radiative transfer, and showed that cloud induced change in upwelling radiation is reduced when $g$ increases. On the other hand, the cloud induced change increases with the extinction of the cloud particles. Both $g$ and $Q_{e}$ were seen to increase as $r_{\text {cryst }}$ deviates from 1 at $664 \mathrm{GHz}$. As a consequence, a compensation effect that reduces brightness temperature sensitivity to variance in $r_{\text {cryst }}$ could hypothetically be attained. For instance, consider a $664 \mathrm{GHz}$ nadir measurement in a cloudy scenario, assuming ice aggregate particles. If $r_{\text {cryst }}$ increases, the resulting increased extinc- tion and asymmetry parameter would result in a positive and negative brightness temperature contribution, respectively, i.e. they cancel each other out to a degree. In other words, $664 \mathrm{GHz}$ observations are potentially even less sensitive to shape. This further suggests the suitability of $664 \mathrm{GHz}$ as a frequency for retrieving ice mass. The effect is not visible for 183 and $325 \mathrm{GHz}$, as $g$ is relatively flat for these frequencies.

On the other hand, it is difficult to make the same argument using the data in the bottom panel of Fig. 8, where $g$ was plotted as a function of $N_{\text {cryst }}$ instead. The cancellation effect discussed in the previous paragraph may be applicable to $N_{\text {cryst }}>300$, where both $g$ and $Q_{e}$ increase with $N_{\text {cryst }}$, but not for $N_{\text {cryst }}<300$. It should also be stressed that $g$ is only investigated using category 2 and 3 data, which are composed of large aggregates only. Satellites measuring at $664 \mathrm{GHz}$ will typically not see these types of large aggregates. In general, these particles are concealed at lower altitudes where $664 \mathrm{GHz}$-channels can not penetrate, unless carried by up-drafts in deep convective systems, for instance. Regardless, for the sake of the $664 \mathrm{GHz}$ channel of ICI, the above arguments are overall promising.

Also interesting is that the spread in bulk extinction coefficient $\left(\gamma_{e}\right)$ and back-scattering coefficient $\left(\gamma_{b}\right)$ narrowed for all frequencies, when $\gamma_{e}$ and $\gamma_{b}$ were plotted as a function of snowfall (Fig. 9). At $183 \mathrm{GHz}$, the spread in $\gamma_{e}$ was reduced by a factor of roughly 2, implying that retrieval of $R_{\text {leq }}$ is less sensitive to shape and can be retrieved with higher accuracy than IWC. Here we consider snowfall throughout the atmosphere; snowfall close to the surface might be secluded from satellite measurements by water vapour extinction. The decrease in spread for $\gamma_{b}$ at $94 \mathrm{GHz}$ was close to 2 as well. This is explained by the fact that high density particles, associated with relatively low aerodynamic areas, tend to have higher fall-speed. This produces a shift rightward in the bottom panels of Fig. 9 and 10 , i.e. the differences in particle fall-speed provides a compensation effect to associated differences in scattering properties. To our knowledge, no previous study has demonstrated this compensatory effect.

The tendencies observed in the triple frequency analysis are qualitatively consistent with previous results (e.g. Kulie et al., 2014; Leinonen and Szyrmer, 2015; Kneifel et al., 2015), where the triple frequency lines are shifted clockwise as the particle effective density or level of riming increases. However, there are differences in magnitude of these shifts and the effective densities that they correspond to; the aggregates of this study seems to be relatively sensitive to $\rho_{\text {eff }}$. On the other hand, the range of DWR-values covered are similar compared to those studies. Otherwise, the $\gamma_{b}$ dependence on particle shape (bottom panel of Fig. 10), at $36 \mathrm{GHz}$ and above, is fairly high. If the objective is to retrieve ice mass or snowfall, this is of course a limitation, while for retrieval of shape information, it is an advantage. Regardless, multi-frequency radar is desirable in order to constrain the retrievals. Radars at 
13.4 GHz and lower (Rayleigh regime), are comparatively insensitive to shape when estimating $R_{\text {leq }}$ (or ice water content).

The external Evans and Tyynelä aggregates, behave somewhat similarly to the aggregates generated in this study, as mentioned previously. They are of fairly high densities (as seen for the Evans aggregate in Fig. 3). As such, they generally produce high $\gamma_{e}$. The Hong aggregate however, having the highest $\rho_{\text {eff }}$-value of all habits, behaves differently. For example, it produces very high extinction when expressed in ice water content, at $183 \mathrm{GHz}$ in the top panel of Fig. 9, but very low extinction at $664 \mathrm{GHz}$ in the bottom panel, when expressed in snowfall rate. The later is an effect of the Hong aggregate being associated with very high fall-speed (small aerodynamic area).

On a final note, above investigations are by no means intended to be a comprehensive study meant to cover all possible aspects of aggregate scattering properties. The use of horizontal random orientation only is a limitation with respect to side-looking satellite radiometers, for instance. Rather, the study serves as a demonstration of the impact of the individual microphysical parameters. Other aggregate parameters were considered as well (maximum diameter, radius of gyration, etc.), but not included as they resulted in similar or insignificant results. It is not fully clear from the analysis what actually causes the observed behaviour in scattering properties (i.e. correlation vs. causation). $r_{\text {cryst }}$ is likely only affecting the scattering properties indirectly, by influencing the overall aggregate shape. The aerodynamic area has a more direct influence, especially when moving into the geometric optics regime. Regardless, $r_{\text {cryst }}$ is not an useful parameter for quantifying real particles, as real aggregates can typically not be defined by a single pristine crystal type. Same argument holds for $N_{\text {cryst }}$. As such, it is promising that both $\rho_{\text {eff }}$ and $A_{\mathrm{r}}$ worked so well as descriptors for the scattering properties.

\section{Conclusions and outlook}

A set of aggregates was generated through simulation in a stochastic and explicit manner. The general characteristics and scattering properties of these aggregates were found to have similarities to other studies, but deviations were also noted. For instance, the $b$-coefficients of the aggregate mass-size relationships were found to be unusually high, and the produced triple frequency signatures are only qualitatively similar to previous studies. The main goal of this study was to explore the response of aggregation and scattering properties to the assumption of crystal shape. The main conclusions are as follows:

- Extinction was generally found to be less sensitive than back-scattering to shape. Active instruments are overall more advantageous for retrieval of snow mass for multiple reasons. However, in this respect passive instruments seem to have an advantage.
- Extinction (both single particle and bulk) at $664 \mathrm{GHz}$ behaves differently compared to the other frequencies. It could be argued that the dependency on shape is relatively low at this frequency, making it suitable for ice mass retrievals particularly.

- Bulk extinction and back-scattering sensitivity to aggregate shape was reduced (by a factor of up to roughly 2) if snowfall rate is considered instead of ice water content. The implication is that retrieval of snowfall rate could be more accurate than for snow water content.

- Calculated triple frequencies saw a clear dependence on crystal axis ratio and conversely the effective density, consistent with both modelling and observational results in the literature. The potential of multifrequency radar measurements for retrieving information on bulk particle properties is thus further established.

- Crystal axis ratio used as input to the aggregation simulation had a high impact on the aggregate effective density and aerodynamic area. The same conclusion holds for the number of constituent crystals (or conversely the crystal mass).

- Crystal axis ratio, constituent crystal number, and the other investigated aggregated parameters have a clear impact on the extinction efficiency, asymmetry parameter, and to a lesser degree, back-scattering efficiency. Aggregate aspect ratio is the exception, to which most single scattering properties were relatively insensitive.

Aforementioned observations may provide some guidance to future ice hydrometeor single scattering databases. The ARTS database (Eriksson et al., 2018) includes aggregates of axis ratios $1 / 6,1.25$, and 5 . However, in order to cover the full extinction span in Fig. 9 for instance, aggregates of axis ratio 15 are required. This type of "needle" aggregate could thus be considered to complement the existing database aggregates well, and will likely be included in a future database version.

\section{Declarations of interest}

None.

\section{Acknowledgements}

Thanks goes to Torbjörn Rathsman, who implemented the snowflake tool-kit as his master thesis project. Also, thanks to Frank Evans and Jani Tyynelä, who provided shape data, and C. D. Westbrook for providing a MATLAB function for the fall-speed calculations. The authors are also grateful to the two anonymous reviewers for their helpful and insightful reviews. The ADDA calculations 
were performed mainly at the central Chalmers cluster, C3SE. Finally, this study was financially supported by the Swedish National Space Board (SNSB) under Grant $150 / 44$.

\section{References}

Baran, A. J., 2009. A review of the light scattering properties of cirrus. J. Quant. Spectrosc. Radiat. Transfer 110 (14-16), 12391260.

Buehler, S. A., Defer, E., Evans, F., Eliasson, S., Mendrok, J., Eriksson, P., C.Lee, Jimenéz, C., Prigent, C., Crewell, S., Kasai, Y. Bennartz, R., Gasiewski, A. J., 2012. Observing ice clouds in the submillimeter spectral range: The CloudIce mission proposal for ESA's Earth Explorer 8. Atmos. Meas. Tech. 5, 1529-1549.

Buehler, S. A., Jiménez, C., Evans, K. F., Eriksson, P., Rydberg, B., Heymsfield, A. J., Stubenrauch, C., Lohmann, U., Emde, C., John, V. O., Sreerekha, T. R., Davis, C. P., 2007. A concept for a satellite mission to measure cloud ice water path and ice particle size. Q. J. R. Meteorol. Soc. 133 (S2), 109-128.

Bühl, J., Alexander, S., Crewell, S., Heymsfield, A., Kalesse, H., Khain, A., Maahn, M., Tricht, K. V., Wendisch, M., 2017. Remote sensing. Meteorol. Monogr. 58, 10.1-10.21.

Ding, J., Bi, L., Yang, P., Kattawar, G. W., Weng, F., Liu, Q. Greenwald, T., 2017. Single-scattering properties of ice particles in the microwave regime: Temperature effect on the ice refractive index with implications in remote sensing. J. Quant. Spectrosc. Radiat. Transfer 190, 26-37.

Draine, B. T., Flatau, P. J., 1994. Discrete-dipole approximation for scattering calculations. J. Optical Soc. o. Am. A 11 (4), 14911499.

Ellis, T. D., L'Ecuyer, T., Haynes, J. M., Stephens, G. L., 2009. How often does it rain over the global oceans? the perspective from cloudsat. Geophys. Res. Lett. 36 (3), 1-5.

Ellison, W. J., 2007. Permittivity of pure water, at standard atmospheric pressure, over the frequency range $0-25 \mathrm{THz}$ and the temperature range $0-100^{\circ}$ c. J. Phys. Chem. Ref. Data. 36 (1), $1-18$

Eriksson, P., Ekelund, R., Mendrok, J., Brath, M., Lemke, O., Buehler, S. A., 2018. A general database of hydrometeor single scattering properties at microwave and sub-millimetre wavelengths. Earth System Science Data 10 (3), 1301-1326.

Eriksson, P., Jamali, M., Mendrok, J., Buehler, S. A., 2015. On the microwave optical properties of randomly oriented ice hydrometeors. Atmos. Meas. Tech. 8 (5), 1913-1933.

Evans, K. F., Wang, J. R., Starr, D. O., Heymsfield, G., Li, L., Tian, L., Lawson, R. P., Heymsfield, A. J., Bansemer, A., 2012. Ice hydrometeor profile retrieval algorithm for high-frequency microwave radiometers: application to the CoSSIR instrument during TC4. Atmos. Meas. Tech. 5 (9), 2277-2306.

Garrett, T. J., Fallgatter, C., Shkurko, K., Howlett, D., 2012. Fall speed measurement and high-resolution multi-angle photography of hydrometeors in free fall. Atmos. Meas. Tech. 5 (11), 2625-2633.

Garrett, T. J., Yuter, S. E., Fallgatter, C., Shkurko, K., Rhodes, S. R., Endries, J. L., 2015. Orientations and aspect ratios of falling snow. Geophys. Res. Lett. 42 (11), 4617-4622.

Geer, A. J., Baordo, F., 2014. Improved scattering radiative transfer for frozen hydrometeors at microwave frequencies. Atmos. Meas. Tech. 7 (6), 1839-1860.

Geer, A. J., Baordo, F., Bormann, N., Chambon, P., English, S. J., Kazumori, M., Lawrence, H., Lean, P., Lonitza, K., Lupu, C. 2017. The growing impact of satellite observations sensitive to humidity, cloud and precipitation. Q. J. R. Meteorol. Soc. 143 (709) 3189-3206

Heymsfield, A., Westbrook, C., 2010. Advances in the estimation of ice particle fall speeds using laboratory and field measurements. J. Atmos. Sci. 67 (8), 2469-2482.

Heymsfield, A. J., Bansemer, A., Field, P. R., Durden, S. L., Stith, J. L., Dye, J. E., Hall, W., Grainger, C. A., 2002. Observations and parameterizations of particle size distributions in deep tropical cirrus and stratiform precipitating clouds: Results from in situ observations in trmm field campaigns. J. Atmos. Sci. 59 (24), 3457-3491.

Heymsfield, A. J., Lewis, S., Bansemer, A., Iaquinta, J., Miloshevich, L. M., Kajikawa, M., Twohy, C., Poellot, M. R., 2002. A general approach for deriving the properties of cirrus and stratiform ice cloud particles. J. Atmos. Sci. 59, 3-29.

Hong, G., 2007. Parameterization of scattering and absorption properties of nonspherical ice crystals at microwave frequencies. J. Geophys. Res. 112 (D11), 11208.

Hong, G., Yang, P., Baum, B. A., Heymsfield, A. J., Weng, F., Liu, Q., Heygster, G., Buehler, S. A., 2009. Scattering database in the millimeter and submillimeter wave range of $100-1000 \mathrm{ghz}$ for nonspherical ice particles. J. Geophys. Res. 114, 6201.

Hou, A. Y., Kakar, R. K., Neeck, S., Azarbarzin, A. A., Kummerow, C. D., Kojima, M., Oki, R., Nakamura, K., Iguchi, T., 2014. The global precipitation measurement mission. Bull. Amer. Met. Soc. 95 (5), 701-722.

Kim, M.-J., 2006. Single scattering parameters of randomly oriented snow particles at microwave frequencies. J. Geophys. Res. 111 (D14), D14201.

Klett, J. D., 1995. Orientation model for particles in turbulence. J. Atmos. Sci. 52 (12), 2276-2285.

Kneifel, S., Kulie, M. S., Bennartz, R., 2011. A triple-frequency approach to retrieve microphysical snowfall parameters. J. Geophys. Res. Atmos. 116 (11), D11203.

Kneifel, S., Lerber, A., Tiira, J., Moisseev, D., Kollias, P., Leinonen, J., 2015. Observed relations between snowfall microphysics and triple-frequency radar measurements. J. Geophys. Res. Atmos. 120 (12), 6034-6055.

Kulie, M. S., Bennartz, R., Greenwald, T. J., Chen, Y., Weng, F., 2010. Uncertainties in microwave properties of frozen precipitation: Implications for remote sensing and data assimilation. J. Atmos. Sci. 67 (11), 3471-3487.

Kulie, M. S., Hiley, M. J., Bennartz, R., Kneifel, S., Tanelli, S., 2014. Triple-Frequency Radar Reflectivity Signatures of Snow: Observations and Comparisons with Theoretical Ice Particle Scattering Models. J. Appl. Meteorol. Clim. 53 (4), 1080-1098.

Kuo, K.-S., Olson, W. S., Johnson, B. T., Grecu, M., Tian, L., Clune, T. L., van Aartsen, B. H., Heymsfield, A. J., Liao, L., Meneghini, R., 2016. The microwave radiative properties of falling snow derived from nonspherical ice particle models. part i: An extensive database of simulated pristine crystals and aggregate particles, and their scattering properties. J. Appl. Meteorol. Clim. 55 (3), 691-708.

Leinonen, J., Kneifel, S., Moisseev, D., Tyynelä, J., Tanelli, S., Nousiainen, T., 2012. Evidence of nonspheroidal behavior in millimeter-wavelength radar observations of snowfall. J. Geophys. Res. Atmos. 117 (D18)

Leinonen, J., Moisseev, D., 2015. What do triple-frequency radar signatures reveal about aggregate snowflakes? J. Geophys. Res. Atmos. 120 (1), 229-239.

Leinonen, J., Szyrmer, W., 2015. Radar signatures of snowflake riming: A modeling study. Earth and Space Science 2 (8), 346-358, 2015EA000102.

Liu, G., 2008. A database of microwave single-scattering properties for nonspherical ice particles. Bull. Amer. Met. Soc. 89, 1563.

Locatelli, J. D., Hobbs, P. V., 1974. Fall speeds and masses of solid precipitation particles. J. Geophys. Res. 79 (15), 2185-2197.

Lu, Y., Jiang, Z., Aydin, K., Verlinde, J., Clothiaux, E. E., Botta, G., 2016. A polarimetric scattering database for non-spherical ice particles at microwave wavelengths. Atmos. Meas. Tech. 9, 51195134 .

Maruyama, K.-i., Fujiyoshi, Y., Maruyama, K.-i., Fujiyoshi, Y., 2005. Monte Carlo Simulation of the Formation of Snowflakes. J. Atmos. Sci. 62 (5), 1529-1544.

Matrosov, S. Y., 2007. Modeling backscatter properties of snowfall at millimeter wavelengths. J. Atmos. Sci. 64 (5), 1727-1736.

Mätzler, C., 2006. Thermal microwave radiation: applications for remote sensing. Vol. 52 of IET electromagnetic waves series. In- 
stitution of Engineering and Technology.

Mie, G., 1908. Beiträge zur optik trüber medien, speziell kolloidaler metallösungen. Annalen der Physik 330 (3), 377-445.

Mishchenko, M. I., Travis, L. D., Lacis, A. A., 2002. Scattering, absorption, and emission of light by small particles. Cambridge University Press, Cambridge, UK.

Mishchenko, M. I., Travis, L. D., Mackowski, D. W., 1996. T-matrix computation of light scattering by nonspherical particles: A review. J. Quant. Spectrosc. Radiat. Transfer 55 (5), 535-575.

Mitchell, D. L., 1996. Use of Mass- and Area-Dimensional Power Laws for Determining Precipitation Particle Terminal Velocities. J. Atmos. Sci. 53 (12), 1710-1723.

O'Shea, S., Choularton, T., Lloyd, G., Crosier, J., Bower, K., Gallagher, M., Abel, S., Cotton, R., Brown, P., Fugal, J., et al., 2016. Airborne observations of the microphysical structure of two contrasting cirrus clouds. J. Geophys. Res. Atmos.

Petty, G. W., Huang, W., 2010. Microwave backscatter and extinction by soft ice spheres and complex snow aggregates. J. Atmos. Sci. 67 (3), 769-787.

Petty, G. W., Huang, W., 2011. The modified gamma size distribution applied to inhomogeneous and nonspherical particles: Key relationships and conversions. J. Atmos. Sci. 68, 1460-1473.

Pruppacher, H. R., Klett, J. D., 1997. Microphysics of Clouds and Precipitation, 2nd Edition. Springer, Dordrecht.

Rathsman, T., 2016. A software toolkit for generating ice and snow particle sharp data. Master's thesis, Msc. thesis, Earth and Space Sciences, Chalmers University of Technology, Gothenburg, Sweden.

URL http://studentarbeten. chalmers. se/publication/234473

Schmitt, C. G., Heymsfield, A. J., 2014. Observational quantification of the separation of simple and complex atmospheric ice particles. Geophys. Res. Lett. 41 (4), 1301-1307.

Sekhon, R. S., Srivastava, R. C., 1970. Snow size spectra and radar reflectivity. J. Atmos. Sci. 27 (2), 299-307.

Stein, T. H. M., Westbrook, C. D., Nicol, J. C., 2015. Fractal geometry of aggregate snowflakes revealed by triple-wavelength radar measurements. Geophys. Res. Lett. 42 (1), 176-183.

Tyynelä, J., Chandrasekar, V., 2014. Characterizing falling snow using multifrequency dual-polarization measurements. J. Geophys. Res. 119 (13), 8268-8283.

Tyynelä, J., Leinonen, J., Moisseev, D., Nousiainen, T., von Lerber, A., 2014. Modeling radar backscattering from melting snowflakes using spheroids with nonuniform distribution of water. J. Quant. Spectrosc. Radiat. Transfer 133, 504-519.

Um, J., McFarquhar, G., Hong, Y., Lee, S., Jung, C., Lawson, R. Mo, Q., 2015. Dimensions and aspect ratios of natural ice crystals. Atmos. Chem. Phys. 15, 3933-56.

Um, J., McFarquhar, G. M., 2009. Single-scattering properties of aggregates of plates. QJRMS 135 (639), 291-304.

Westbrook, C., Ball, R., Field, P., Heymsfield, A. J., 2004. Theory of growth by differential sedimentation, with application to snowflake formation. Phys. Rev. E 70 (2), 021403.

Yin, M., Liu, G., Honeyager, R., Joseph Turk, F., 2017. Observed differences of triple-frequency radar signatures between snowflakes in stratiform and convective clouds. J. Quant. Spectrosc. Radiat. Transfer 193, 13-20.

Yurkin, M. A., Hoekstra, A. G., 2011. The discrete-dipoleapproximation code adda: capabilities and known limitations. J. Quant. Spectrosc. Radiat. Transfer 112 (13), 2234-2247. 